\title{
Ray-Tracing Simulations Characterising the Performance of the Proposed HFIR HB4 Main Shutter
}

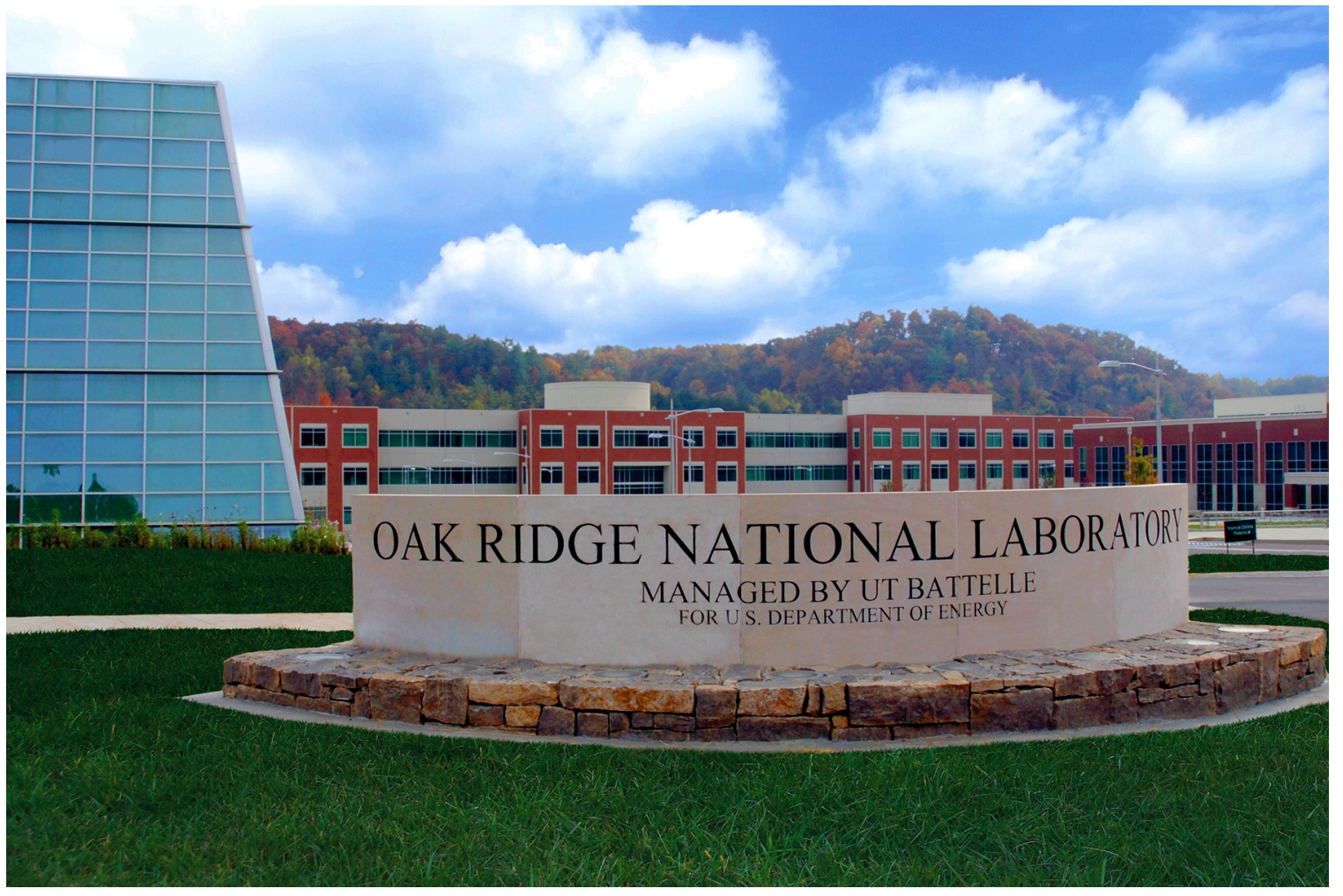

Matthew J. Frost

Garrett Granroth

Thomas Huegle

J. Lee Robertson

August 2021 


\section{DOCUMENT AVAILABILITY}

Reports produced after January 1, 1996, are generally available free via US Department of Energy (DOE) SciTech Connect.

Website: www.osti.gov/

Reports produced before January 1, 1996, may be purchased by members of the public from the following source:

National Technical Information Service

5285 Port Royal Road

Springfield, VA 22161

Telephone: 703-605-6000 (1-800-553-6847)

TDD: $703-487-4639$

Fax: 703-605-6900

E-mail: info@ntis.gov

Website: http://classic.ntis.gov/

Reports are available to DOE employees, DOE contractors, Energy Technology Data Exchange representatives, and International Nuclear Information System representatives from the following source:

Office of Scientific and Technical Information

PO Box 62

Oak Ridge, TN 37831

Telephone: 865-576-8401

Fax: 865-576-5728

E-mail: report@osti.gov

Website: http://www.osti.gov/contact.html

This report was prepared as an account of work sponsored by an agency of the United States Government. Neither the United States Government nor any agency thereof, nor any of their employees, makes any warranty, express or implied, or assumes any legal liability or responsibility for the accuracy, completeness, or usefulness of any information, apparatus, product, or process disclosed, or represents that its use would not infringe privately owned rights. Reference herein to any specific commercial product, process, or service by trade name, trademark, manufacturer, or otherwise, does not necessarily constitute or imply its endorsement, recommendation, or favoring by the United States Government or any agency thereof. The views and opinions of authors expressed herein do not necessarily state or reflect those of the United States Government or any agency thereof. 


\title{
Ray-Tracing Simulations Characterising the Performance of the Proposed HFIR HB4 Main Shutter
}

\author{
Matthew J. Frost \\ Garrett Granroth \\ Thomas Huegle \\ J. Lee Robertson
}

August 2021

Prepared by OAK RIDGE NATIONAL LABORATORY

Oak Ridge, TN 37831-6283

managed by

UT-Battelle LLC

for the

US DEPARTMENT OF ENERGY

under contract DE-AC05-00OR22725 



\section{CONTENTS}

LIST OF FIGURES $\ldots \ldots \ldots \ldots \ldots \ldots \ldots \ldots \ldots \ldots$

LIST OF TABLES $\ldots \ldots \ldots \ldots \ldots \ldots \ldots \ldots \ldots$ vii

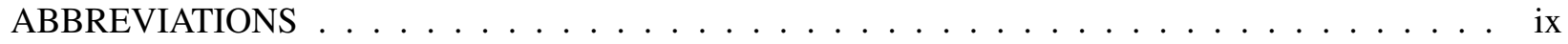

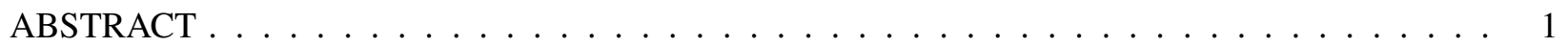

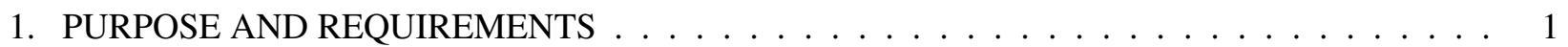

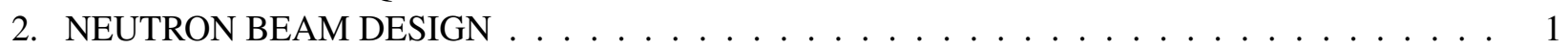

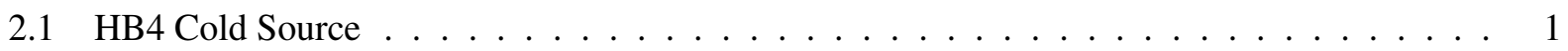

2.2 HB4 Beamtube . . . . . . . . . . . . . . . . . . . . . 2

2.3 HB4 Main Shutter Guide Channels . . . . . . . . . . . . . . . . . . . . . 2

2.3.1 Guide Channel Shape and Reflectivity . . . . . . . . . . . . . . . . . . . 2

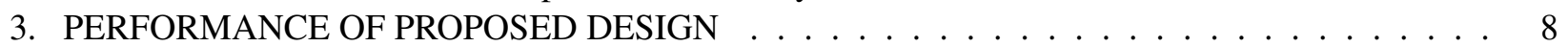

4. ALIGNMENT REQUIREMENTS OF PROPOSED DESIGN . . . . . . . . . . . . . . . . . 11

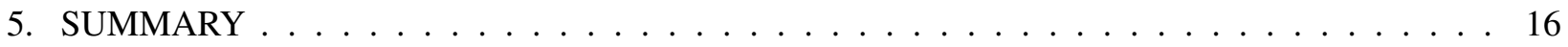

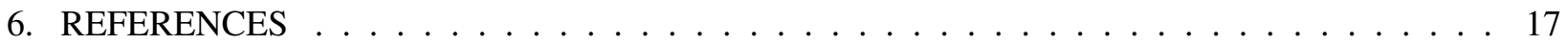

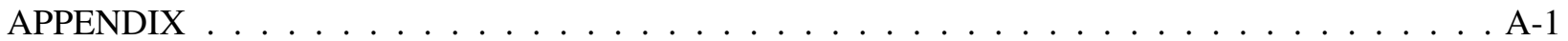

A CORRECTION TO 2007 HB4 COLD SOURCE BRIGHTNESS MEASUREMENT . . . . . . . A-2

B MCSTAS CODE REPRESENTING THE COLD SOURCE AND BEAMTUBE AT HB4 . . . . . A-5

C MCSTAS CODE REPRESENTING THE MAIN SHUTTER AT HB4 . . . . . . . . . . . . . . A-14

D MAIN SHUTTER GUIDE CHANNEL S1 OFF FILE . . . . . . . . . . . . . . . . . . A A-19

E Main Shutter Guide Channel S2 OFF File . . . . . . . . . . . . . . . . . . . . . . A A-20

F MAIN SHUTTER GUIDE CHANNEL S3 OFF FILE . . . . . . . . . . . . . . . . . . . . A-21 



\section{LIST OF FIGURES}

1 A schematic layout showing the planned guide start interface geometry at the exit of the Main Shutter. . . . . . . . . . . . . . . . . . . . . . 4

2 A cross-cut view of the cold source within the HB4 Beam tube. . . . . . . . . . . . . . 4

3 Plots describing the HB4 cold source used in McStas simulations. . . . . . . . . . . . . 5

4 An overhead view of the simulated beamtube and collimator in McStas. . . . . . . . . . 5

5 A view of the available neutron flux at the exit of the internal collimator. . . . . . . . . 6

6 A not-to-scale over-head view of the proposed Guide Channel geometry for the HB4

Main Shutter. . . . . . . . . . . . . . . . . . . . . . 6

7 A plot describing the required reflectivity cut off for each channel and instrument. . . . . . 7

8 A plot showing the spectral neutron flux at the exit to the shutter. . . . . . . . . 8

9 A plot showing the effect increasing the $m$-value has on the average exit flux. . . . . . . 9

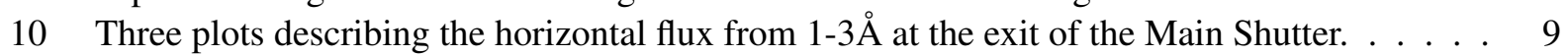

11 A scan of the $m$ value for each of the three guide channels. . . . . . . . . . . . 10

12 A diagram showing the typical coordinate system utilized in most neutron ray tracing

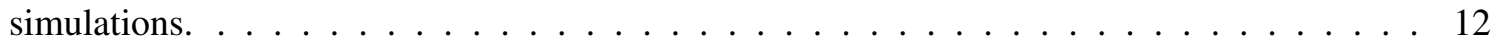

13 Plots describing the effect of pitch misalignment on NB6 spectrum. . . . . . . . . . . . 12

14 Plots describing misalignment effects for the whole instrument suite . . . . . . . . . . . 13

15 Plots describing misalignment effects for the NB1 Beam Guide. . . . . . . . . . . . . . 13

16 Plots describing misalignment effects for the NB2 Beam Guide. . . . . . . . . . . . . . 13

17 Plots describing misalignment effects for the NB3 Beam Guide. . . . . . . . . . . . . . . 14

18 Plots describing misalignment effects for the NB4 Beam Guide. . . . . . . . . . . . . . . 14

19 Plots describing misalignment effects for the NB5 Beam Guide. . . . . . . . . . . . . . . . 14

20 Plots describing misalignment effects for the NB6 Beam Guide. . . . . . . . . . . . . . . 15

21 The simulated, measured and fitted brightness from the HFIR cold source in 2007. . . . . . . A-2

22 A schematic describing the layout of the brightness measurement and associated spectrum. . A-3

23 A schematic describing acceptance determination via Monte Carlo simulation. . . . . . . . . A-4 



\section{LIST OF TABLES}

1 The neutron spectral brightness is defined using a triple Maxwell-Boltzmann Distribution

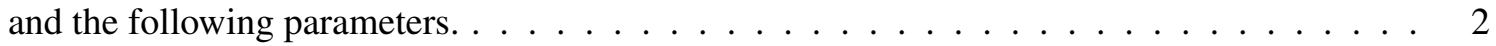

2 The baseline geometry and reflectivity for the Main Shutter guide channels can be determined via the Wavelength and Divergence requirements for each instrument [1]. . . . . 2

3 The analytically determined and proposed $\mathbf{m}$ values for the Main Shutter Guide Channels $\mathrm{S} 1, \mathrm{~S} 2$, and $\mathrm{S} 3 . \ldots \ldots \ldots \ldots \ldots \ldots \ldots \ldots$

4 A table of values interpreting the simulated losses and plots for each beam guide. . . . . . 16 



\section{ABBREVIATIONS}

ORNL Oak Ridge National Laboratory

HFIR High Flux Isotope Reactor

HBRR HFIR Beryllium Reflector Replacement

FOM Figure of Merit

ROI Region of Interest

MCNP Monte Carlo N-Particle 



\begin{abstract}
The Main Shutter at HB4 will serve two purposes after the HFIR Beryllium Reflector Replacement planned to take place in 2024. First as the primary certified safety control controlling the passage of neutrons from the cold source in the HFIR pressure vessel into the cold guide hall, and second as the first set of reflecting surfaces used to guide neutrons from the source and into the individual guide starts for each instrument in the cold guide hall.
\end{abstract}

\title{
1. PURPOSE AND REQUIREMENTS
}

As stated above, the main shutter will contain the first set of reflecting surfaces serving any of the instruments in the cold hall after 2024. The goal of the reflecting surfaces at this location is to enhance the total cold neutron flux available to any of those instruments. As a result, the guide geometry and coatings are especially tailored to meet the needs of the planned instruments in the cold guide hall upgrade. Furthermore, the positioning and alignment requirements for this multiplexed guide section within the main shutter body will need to meet standards as determined by simulating misalignment of the guide section. This will be the main result of this report.

\section{NEUTRON BEAM DESIGN}

The simulation software utilized to understand the proposed geometry and its alignment requirements is McStas [2]. McStas provides a straightforward means by which to replicate the anticipated performance of the HFIR cold source and the instruments that utilize its neutron flux. This analysis comprises three distinct assemblies: the Cold Source, the Beam Tube and the Main Shutter. Within the Main Shutter assembly are three guide channels, S1, S2, and S3. S1 will feed NB1, NB3, and NB4. S2 will feed NB2 and NB5. S3 will feed NB6. The planned layout at the exit of the shutter is seen in Figure 1. Surface reflectivity will be optimized to accommodate the instrument with the most demanding requirements in each channel.

\subsection{HB4 Cold Source}

The HB4 Cold source is liquid hydrogen at $20 \mathrm{~K}$ and about 15 atm pressure confined within a volume of $465 \mathrm{~cm}^{3}$ [3]. The viewable surface of the source is nominally an oval with minor radius $3 \mathrm{~cm}$ and major radius $4 \mathrm{~cm}$, providing an emission area of approximately $40 \mathrm{~cm}^{2}$. This oval geometry is due to the inlet and outlet ports needed to circulate the hydrogen through the beam tube and into the source volume. As seen in Figure 2, the location of the cold source is at the deepest possible location within the existing beam tube design.

Detailed simulations describing the overall performance of the source can be found in [3] as well as a survey of possible future improvements. The McStas simulation uses the component Source_gen in combination with masks to replicate the emission area and spectral shapes of the cold source. As seen in Figure 3, the total emitted area from the source is $39.53 \mathrm{~cm}^{2}$. The spectrum emitted by this area is that of a triple Maxwell-Boltzmann distribution using the parameters in Table 1 to dictate the integrated source brightness and temperature of each. These were determined from [4] and revised downward by $17 \%$ in 2020. The details of this revision can be seen in Appendix A. The spectrum used to replicate the measurement is seen in figure 3 . 
Table 1. The neutron spectral brightness is defined using a triple Maxwell-Boltzmann Distribution and the following parameters.

\begin{tabular}{cc}
\hline $\begin{array}{c}\text { Brightness } \\
\left.\text { [neutrons } /\left(\mathbf{c m}^{2} \cdot \mathbf{s r} \cdot \mathbf{s}\right)\right]\end{array}$ & $\begin{array}{c}\text { Temperature } \\
\text { [Kelvin] }\end{array}$ \\
\hline \hline $6.07 \times 10^{12}$ & 325 \\
$2.61 \times 10^{13}$ & 67.2 \\
$7.95 \times 10^{12}$ & 27.3 \\
\hline
\end{tabular}

\subsection{HB4 Beamtube}

The HB4 Beam Tube contains many components, most of which are used to support the cold source volume deep inside the tube and provide initial coarse collimation of the neutron flux in the beam tube. Figure 4 shows an over-head view of the source, beam obstructions and the collimator as described by the McStas simulations provided in Appendix B.

\subsection{HB4 Main Shutter Guide Channels}

The Main Shutter sits approximately $80 \mathrm{~cm}$ downstream of the exit of the primary collimator. This shutter will be a large cylinder that rotates about a vertical axis. This will provide both radiological protection when closed and a sturdy housing for the guide channels to reside. Since the shutter will be movable and contain a key optical component, the alignment of the shutter when initially installed will be very important. Additionally, the alignment of the shutter drive end point in the open position is also crucial to the performance of the guide as will be shown in Section 4.

\subsubsection{Guide Channel Shape and Reflectivity}

As described prior, there are three channels in the Main Shutter Guide insert. These are the first reflecting surfaces for the instruments that are proposed for the Cold Guide hall upgrade. The optical requirements within the shutter are determined by what the source can provide through the primary collimator and what the instruments need to meet their science goals. Those needs are dictated by the minimum neutron wavelength and maximum beam divergence on the sample, as noted in [1]. A summary of these requirements is seen in Table 2.

Table 2. The baseline geometry and reflectivity for the Main Shutter guide channels can be determined via the Wavelength and Divergence requirements for each instrument [1].

\begin{tabular}{lccl}
\hline Instrument & $\begin{array}{c}\text { Shutter } \\
\text { Channel }\end{array}$ & $\begin{array}{c}\text { Min. Wavelength } \\
\text { [Angstroms] }\end{array}$ & $\begin{array}{l}\text { Max. Divergence VxH } \\
\text { [FWHM Degrees] }\end{array}$ \\
\hline \hline NB1 IMAGINE & S1 & 2.0 & $0.2 \times 0.2$ \\
NB2 NSE/Alignment & S2 & 2.4 & $1.5 \times 0.9$ \\
NB3 BioSANS & S1 & 3.0 & $0.5 \times 0.5$ \\
NB4 Imaging & S1 & 2.4 & $0.4 \times 0.4(\mathrm{~L} / \mathrm{D} 150)$ \\
NB5 GPSANS & S2 & 3.0 & $0.5 \times 0.5$ \\
NB6 MANTA & S3 & 1.8 & $3.0 \times 2.0$ \\
\hline
\end{tabular}


Based on these requirements, combined with the location of the Main Shutter and the phase space permitted by the beamtube collimator, one can layout a geometry that will meet almost all of the instrument requirements seen in Table 2. The assignments of each instrument to a certain channel was based on layout in the guide hall and sensitivity to guide curvature disruptions; details regarding this feature of the instrument layout are documented in [1]. One can see the collimator exit flux distribution and source to shutter distance in Figure 5, which dictates the permitted range of guide channels and allowed angular acceptance. From this, an entrance/exit geometry (not-to-scale) is proposed and seen in Figure 6 . The geometry permits angles of 1.39, 1.39, and 1.53 degrees of horizontal acceptance for S1, S2 and S3 respectively. S1 and S2 meet their horizontal divergence requirements nicely, and while S3 does not, the flux distribution requirement of $20 \mathrm{~mm}$ will provide the flexibility needed to convert the wider space distribution into a wider beam divergence using the right focusing optic design. Vertically, the source is larger and the openings into each guide is equal to or greater than any of the widths, thus any concern trying to fill the required vertical phase space is minimal.

Using these angles, one can then analytically determine the super-reflective coating cut-off, $m$, needed to reflect a given neutron wavelength $\lambda$ at the maximum required angle $\theta$. The equation dictating this is

$$
m=10 \frac{\theta\left[^{\circ}\right]}{\lambda[\AA]}
$$

Figure 7 plots this equation for each instrument and guide channel.

The geometry used to describe all three guide channels is given in Appendices D, E, and F in the form of OFF geometry files. 


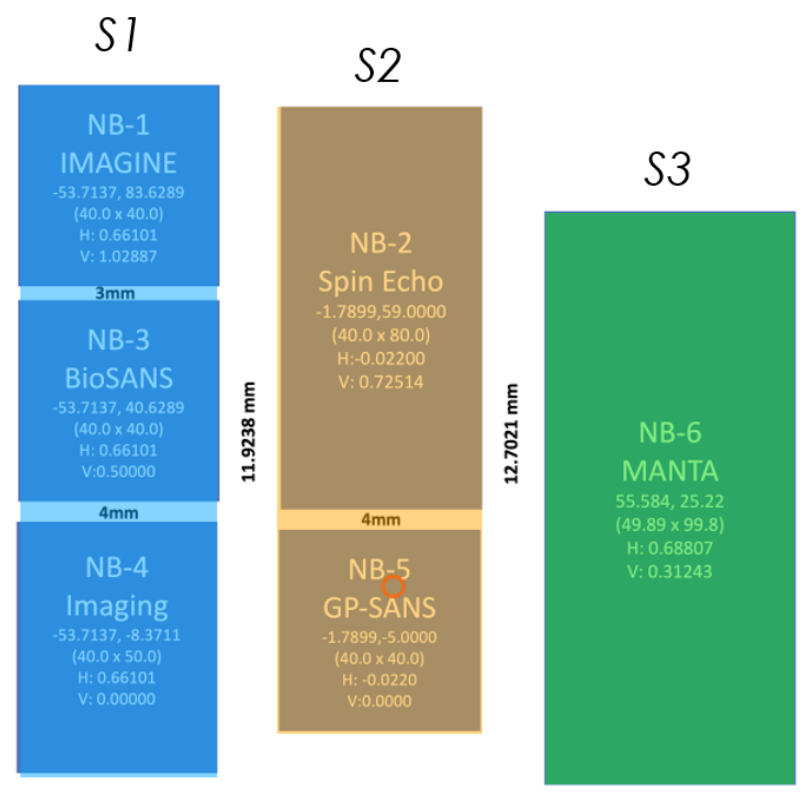

Figure 1. A schematic layout showing the planned guide start interface geometry at the exit of the Main Shutter.

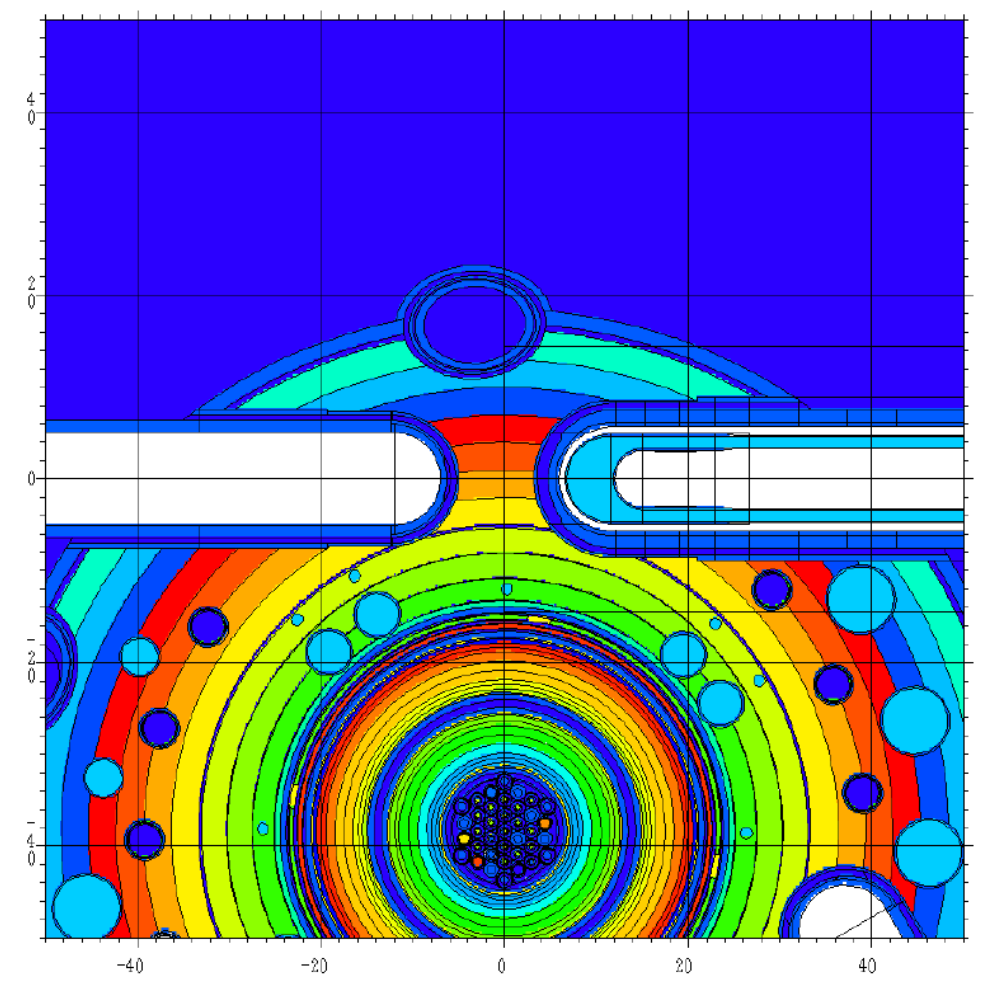

Figure 2. A cross-cut view of the cold source within the HB4 Beam tube. The cold source resides at the deepest position possible in the HB4 beamtube and contains $465 \mathrm{~cm}^{3}$ of liquid hydrogen. More details can be found in [3]. 

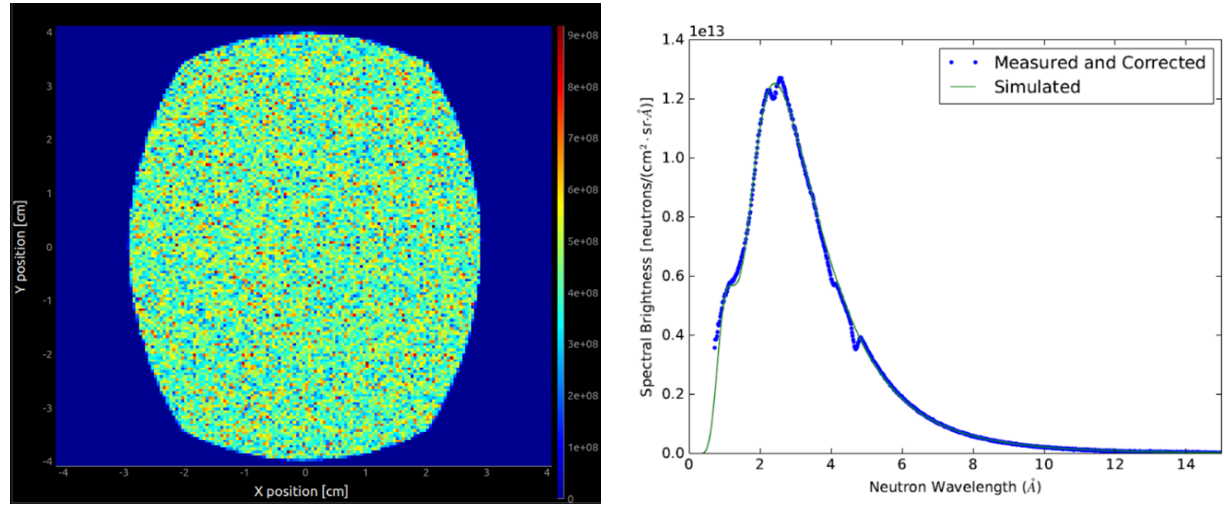

Figure 3. Plots describing the HB4 cold source used in McStas simulations. [Left] An image of the cold source flux distribution. [Right] The spectral brightness of the HB4 cold source. The blue dots are measured [4] and the green trace is the simulated spectrum from Triple MB fit using parameters seen in Table 1.

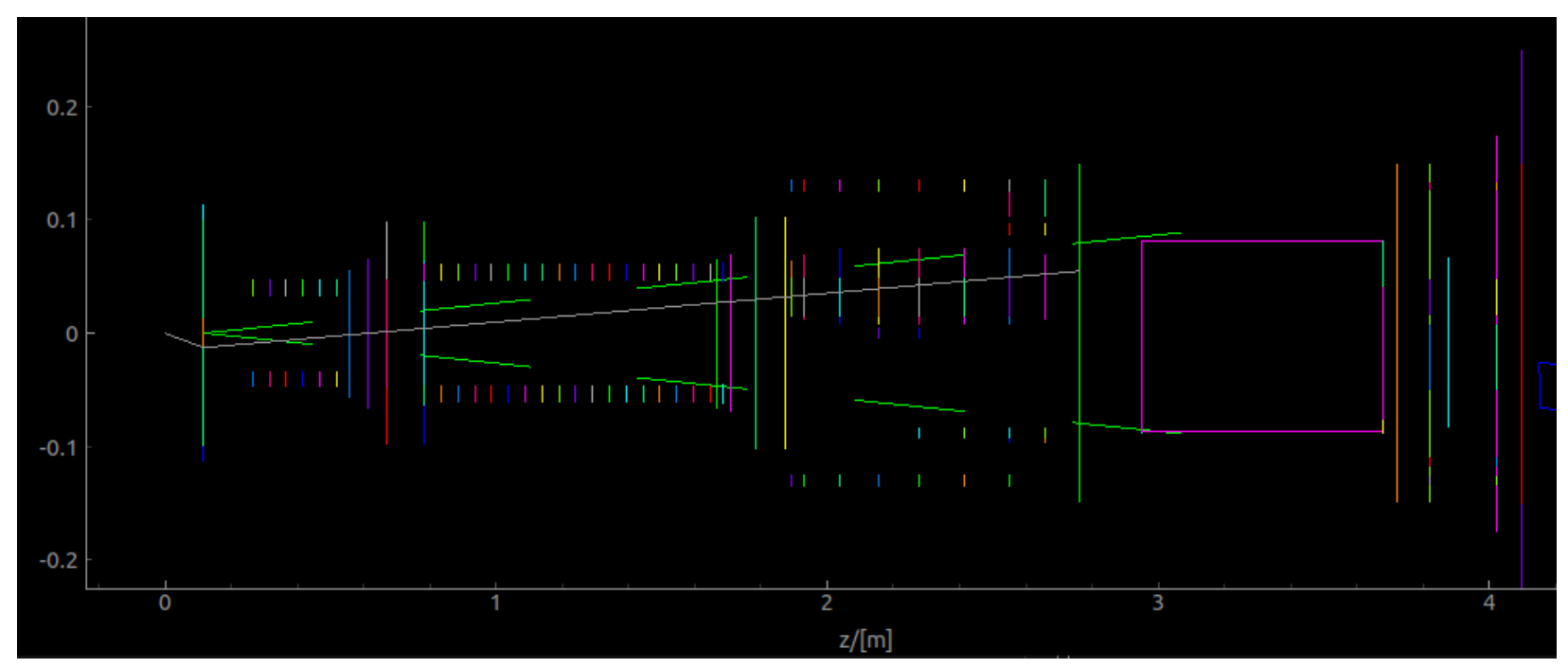

Figure 4. An overhead view of the simulated beamtube and collimator in McStas. The neutron flux comes from the left, and is projected on to the entrance of the collimator (magenta). 


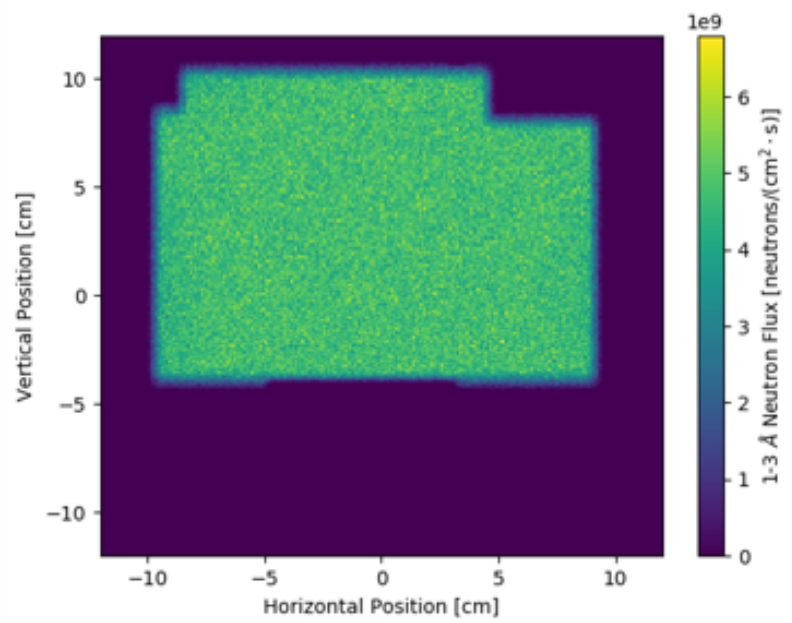

Figure 5. A view of the available neutron flux at the exit of the internal collimator [Top]. An overhead view of the source, collimator and proposed Main Shutter Guide Channel surfaces [Bottom].

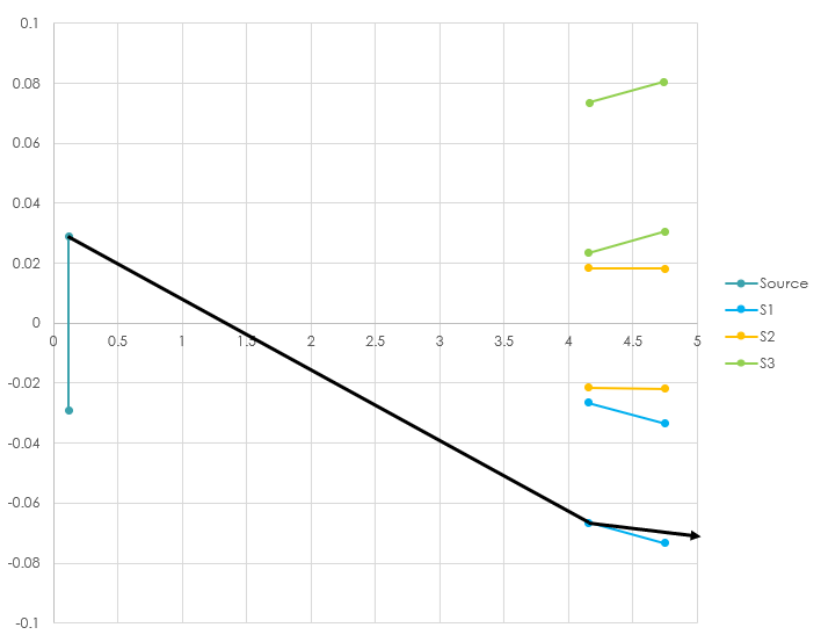

Figure 6. A not-to-scale over-head view of the proposed Guide Channel geometry for the HB4 Main Shutter. The black line is an example of a neutron trajectory that would interact with the shutter channel geometry. The source and shutter location combined with their geometry dictate the angular acceptance of the guide channels. 


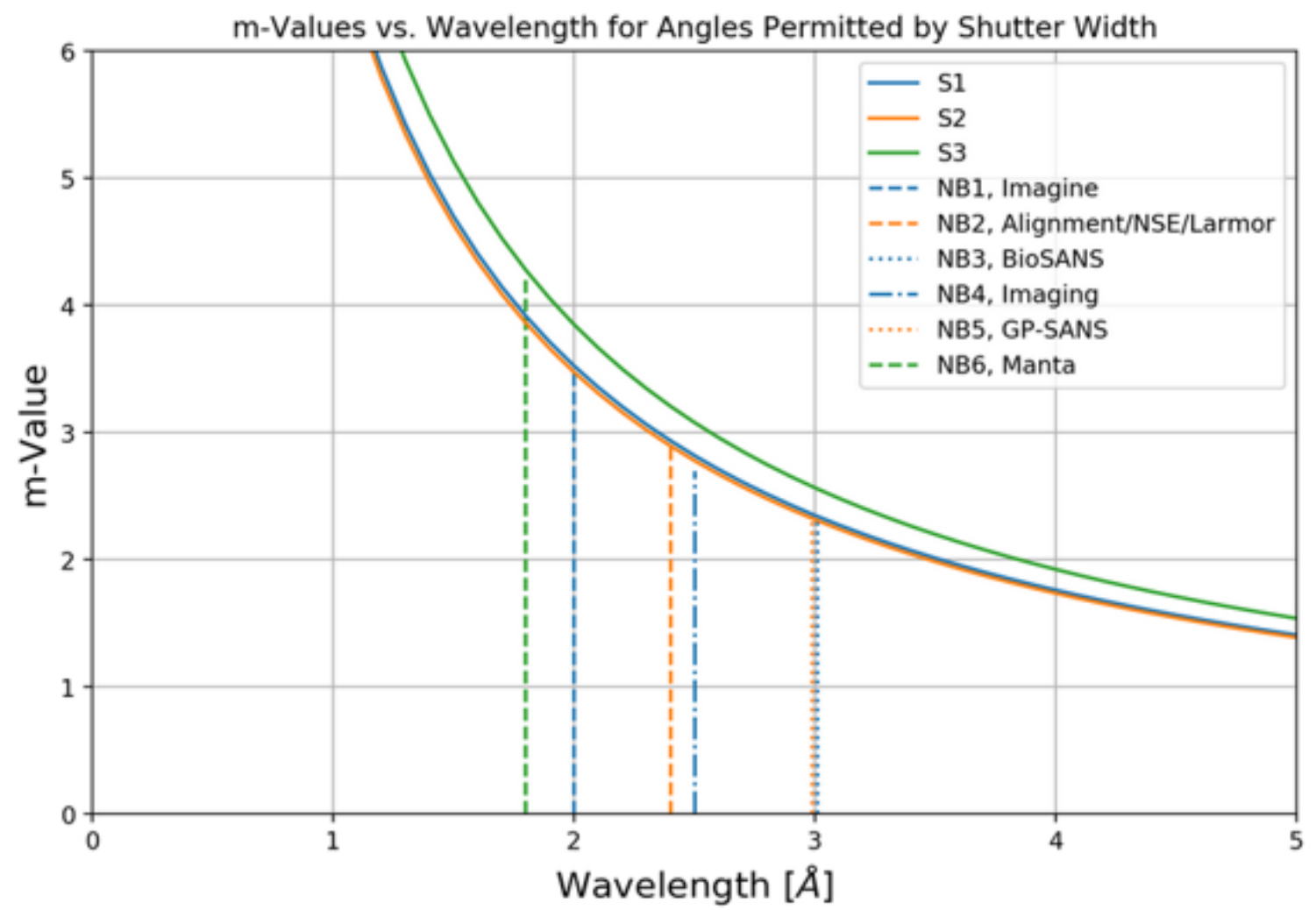

Figure 7. A plot describing the required reflectivity cutoff for each channel and instrument. The decay traces are from Equation 1, and the vertical lines represent the minimum required wavelength. 


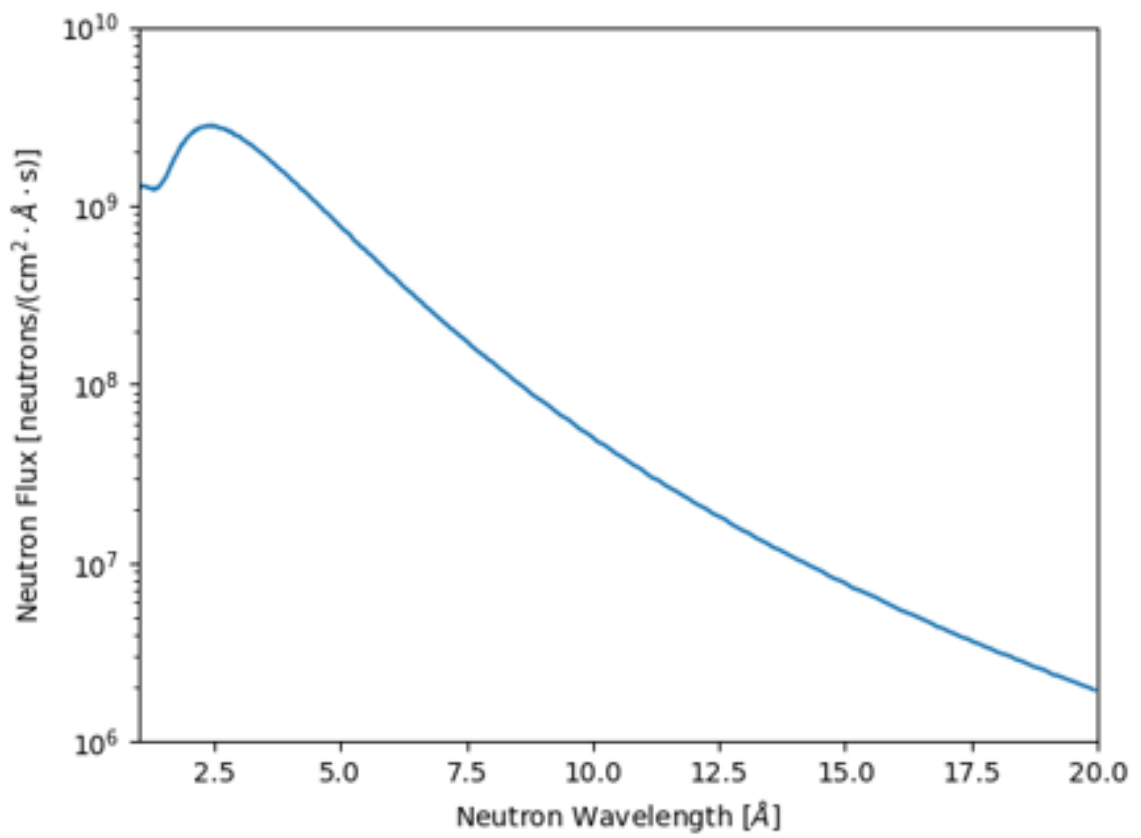

Figure 8. A plot showing the spectral neutron flux at the entrance to the shutter.

\section{PERFORMANCE OF PROPOSED DESIGN}

The previous section provided a calculation of the required surface reflectivity based strictly on the horizontal acceptance and minimum wavelength required for the instruments. This section will show that while the analytical calculation is correct, the value added for higher $m$ values on the channels is not substantial in the wavelengths of interest. This will be done using McStas ray-tracing simulations. From neutron wavelengths $1-20 \AA$ there is $8 \times 10^{9}$ neutrons $/\left(\mathrm{cm}^{2} \cdot \mathrm{s}\right)$ flux available at the entrance to the shutter, and fully illuminates all three channels. An area-normalized spectrum can be seen in Figure 8.

All three guide surfaces provide a significant increase in the flux at the edges of the exit aperture. This is expected, as low angles will reflect and transport better than the higher incident angles, if any. The result is a notable increase in the average flux density with increased super-mirror cutoff, $m$, especially at shorter wavelengths, as seen in Figure 9. Locally, the enhancement in flux can be seen in Figure 10. The same effect is noted across all three guide channels, and even more so in guide channel S3.

While the increase in flux is beneficial, the value added by utilizing higher $m$ values in these guide channels is not immediately obvious. Figures 9 and 10 show this to some extent, but the improvement is better realized with a fine scan of the $m$ value for each guide channel over a shorter wavelength range. Figure 11 shows the change in the average flux for $m=1$ to $m=5$ for all three channels.

Given Figure 11, it is clear that while the improvement of the flux at the guide exit is notable up to $m=3$, it is not substantial for $m$ values beyond for S1 and S2. Thus, $m=3$ is the best suited reflectivity cutoff for these channels. S3 does benefit beyond $m=3$ in this wavelength range, due mainly to the fact that its geometry permits a wider acceptance and NB6 utilizes a shorter wavelength. The conclusion is the best $m$ value for S3 is $m=3.5$. These values, as well as the analytically determined $m$ values from Section 2.3.1 can be seen in Table 3 . 

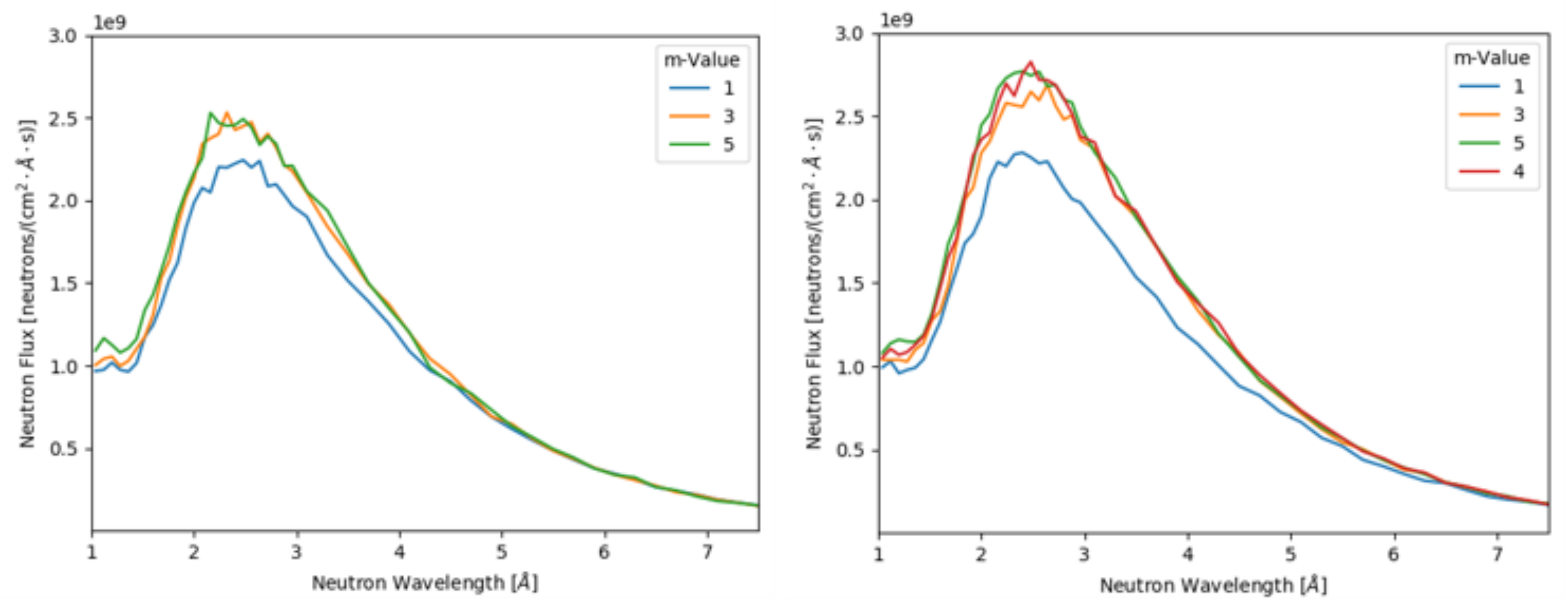

Figure 9. A plot showing the effect increasing the m-value has on the average exit flux. [Left] A scan of $\mathrm{S} 1$ supermirror coating shows an improvement in average flux, but very little above $\mathrm{m}=3$. [Right] The scan for S3 showing a higher average flux gain and benefit for m-value up to 4 .
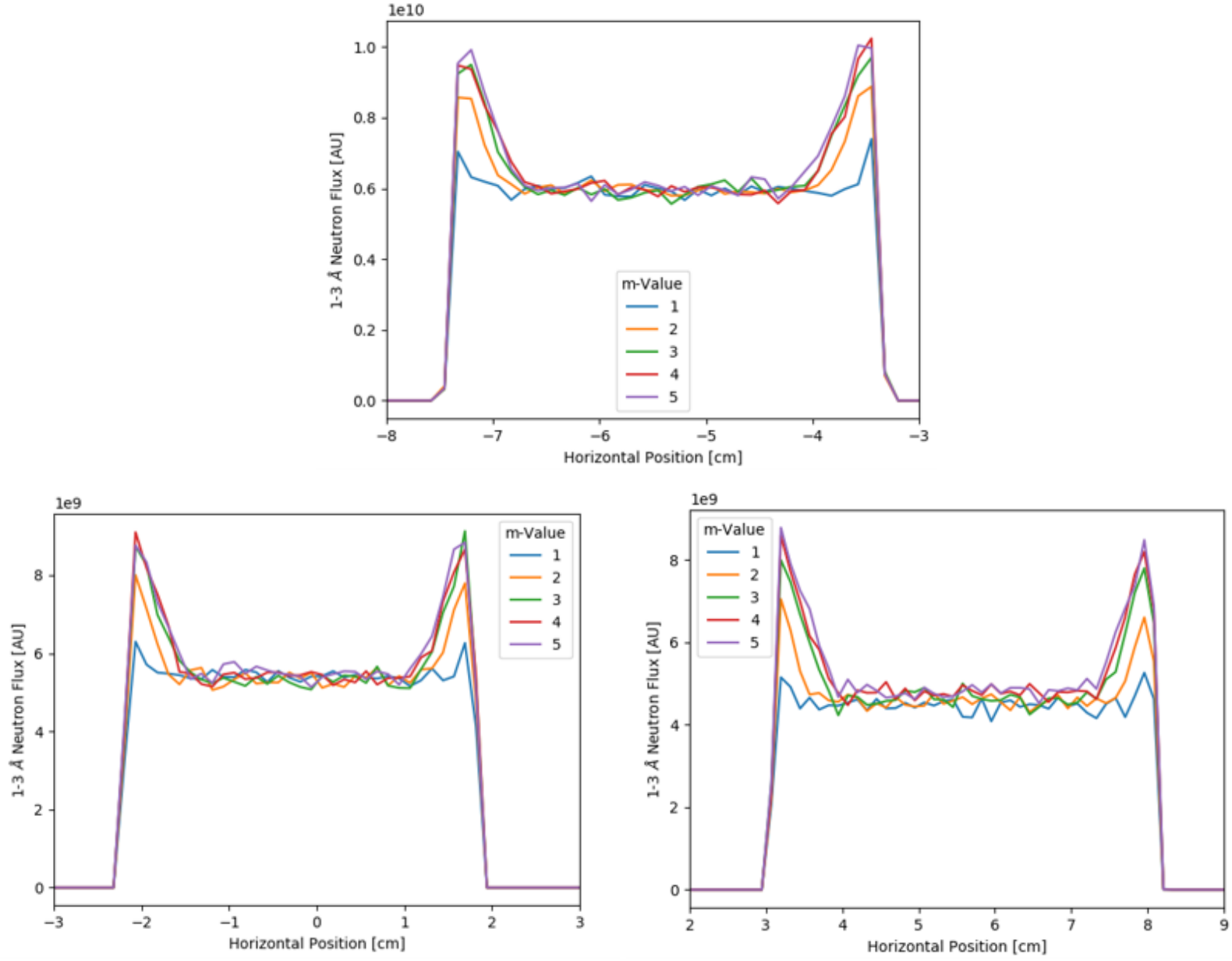

Figure 10. Three plots describing the horizontal flux from 1-3 $\AA$ at the exit of the Main Shutter. The flux is plotted for a range of $m$ values for S1 [Top], S2 [Left], and S3 [Right]. 


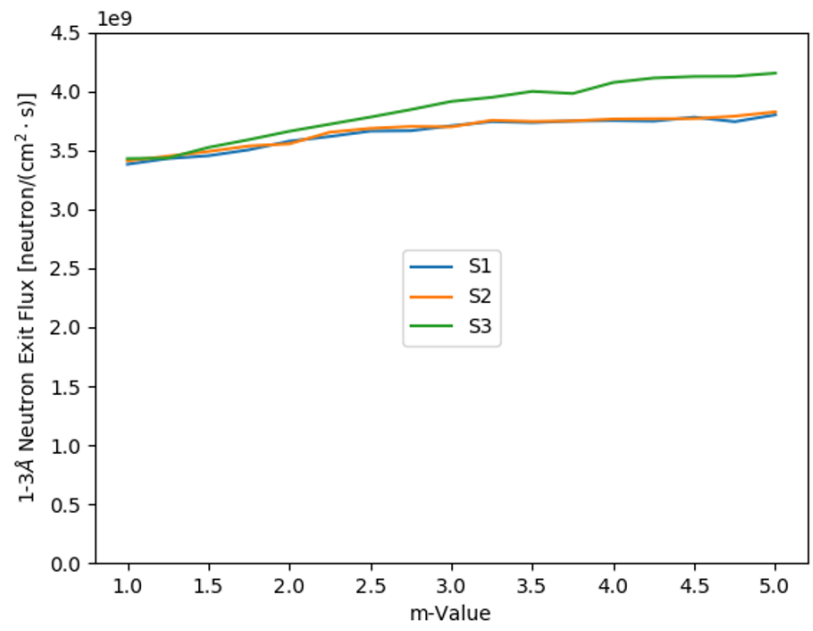

Figure 11. A scan of the $\mathbf{m}$ value for each of the three guide channels. The improvement in the average $1-3 \AA$ flux at the guide channel exit is limited to $m=3$ for S1 and S2, and $m=4$ for S3.

Table 3. The analytically determined and proposed $m$ values for the Main Shutter Guide Channels S1, S2, and S3.

\begin{tabular}{ccc}
\hline Guide Channel & $\begin{array}{c}\text { Analytical } \\
\text { m-value }\end{array}$ & $\begin{array}{c}\text { Optimized } \\
\text { m-value }\end{array}$ \\
\hline \hline S1 & 3.5 & 3.0 \\
S2 & 3.0 & 3.0 \\
S3 & 4.2 & 3.5 \\
\hline
\end{tabular}




\section{ALIGNMENT REQUIREMENTS OF PROPOSED DESIGN}

It has been shown in the previous sections that the guide channels proposed for the HB4 shutter will provide a notable boost in intensity and beam divergence available to the instrument suite in the cold guide hall. However, their performance is very dependent on the alignment of these initial reflecting features. The guide channels will be inside of a cylinder that rotates about a vertical axis to either a closed or open position.

The closed position will align the channels such that they permit no neutron beam into the guide hall, and the open position will be precisely aligned such that the boost in flux provided by the upstream reflecting surfaces is effectively transported into the rest of the guide system.

Simulations were performed in order to quantify the impact any misalignment would have on the intensity provided to the downstream guide system. These simulations used a modified version of the code found at https://code.ornl.gov/sns-neutronics/mcstas-wg/hb4-cold-source-2024. The modifications added an aperture and spectrum monitor for each proposed guide start just downstream of the guide channel exit. This aperture and monitor replicate the expected acceptance of those guide starts, and will be used as input to the Figure-of-Merit (FOM) to understand the relative impact due to misalignment of the guide channels. The FOM to be used for this simulation will be the relative intensity of the monitor as compared to perfect alignment in two different wavelength regions. As seen in the Figure 13, scanning the pitch of of the guide channel from $-0.40^{\circ}$ to $0.35^{\circ}$ has a substantial effect on the relative intensity, but the impact is different at shorter wavelengths. Thus, the FOM is divided into short and long wavelength FOM's and the performance quantified across the scan range. Each beam guide start was scanned in six degrees of freedom, with each position dimension scanned from $+/-4.0$ millimeters and rotation dimensions scanned over a range $+/-0.4^{\circ}$.

Misalignment in three of the dimensions has the same nominal effect across the whole suite. These are along the $\mathrm{z}$-axis (along the beam), the x-axis (transverse horizontal to the beam), and roll (rotation about the z-axis). The effects can be seen in Figure 14. One notes that misalignment along the beam and around the beam axis is of little consequence, but misalignment along the transverse horizontal direction is substantial, with almost a $3 \%$ loss per millimeter of misalignment.

The impact that misalignment has on the rest of the dimensions varies greatly between the guide starts. Plots showing the impact of vertical, pitch and vertical axis rotation misalignment can be seen in Figures $15,16,17,18,19$ and 20 for NB1, 2, 3, 4, 5, and 6 respectively. 


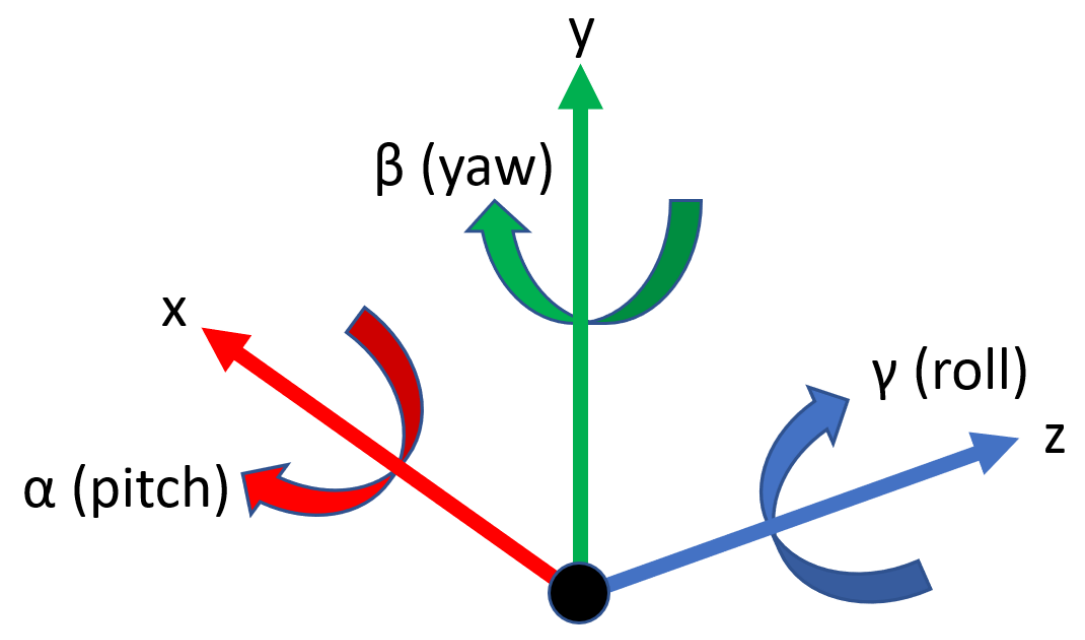

Figure 12. A diagram showing the typical coordinate system utilized in most neutron ray tracing simulations.
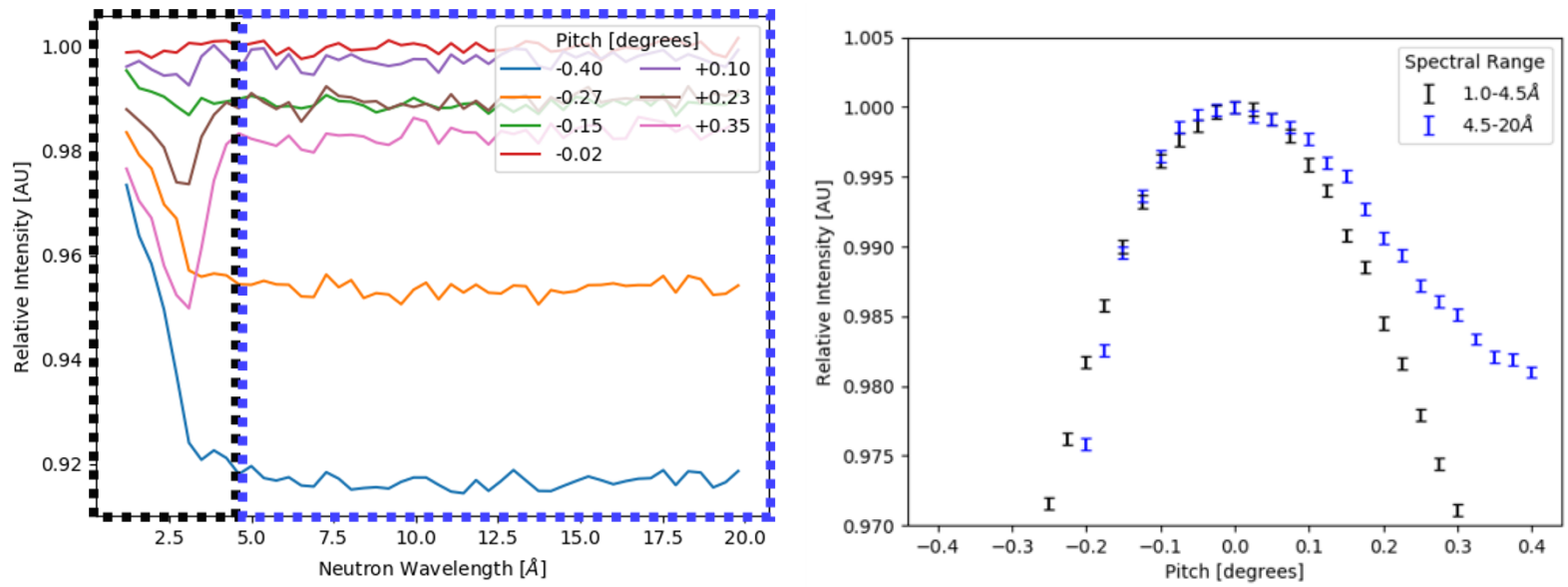

Figure 13. Plots describing the effect of pitch misalignment on NB6 spectrum. [Left] The relative spectral intensity across a range of pitch orientations for S3. [Right] The degradation in performance across the short and long wavelength regions of interest versus pitch angle. The ROI's shown the left plot correlate to data points seen in the right plot. 

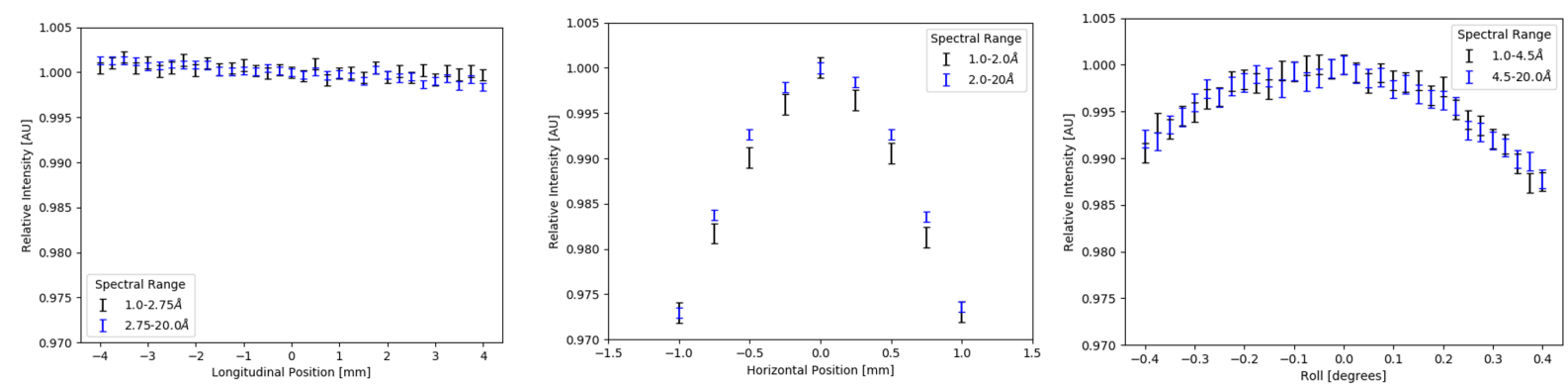

Figure 14. Plots describing misalignment effects for the whole instrument suite. [Left] The relative intensity impact of misalignment along the nominal beam trajectory. [Middle] The relative intensity impact of misalignment transverse horizontal to the beam. [Right] The relative intensity impact of angular misalignment around the z-axis.
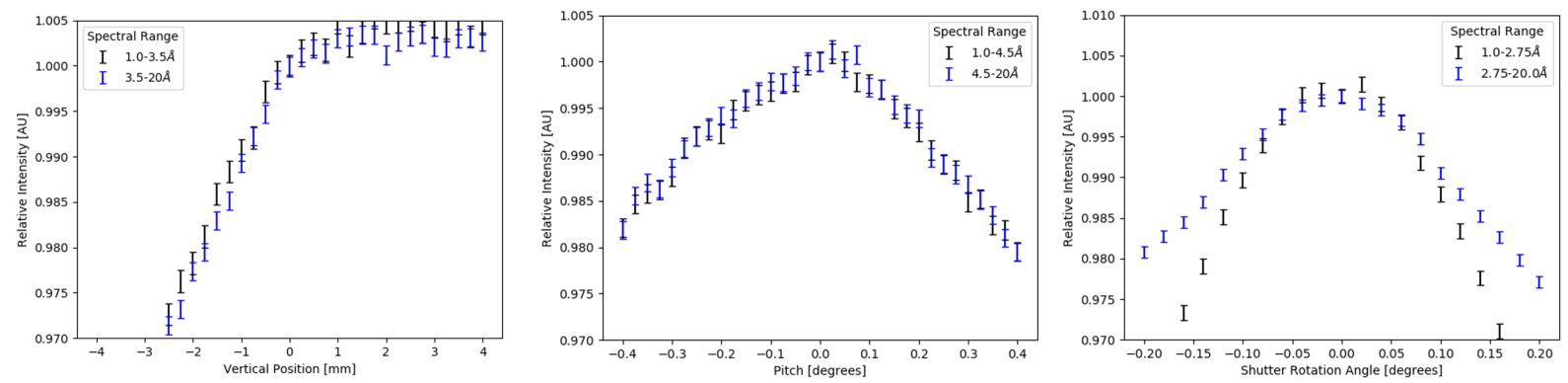

Figure 15. Plots describing misalignment effects for the NB1 Beam Guide. [Top] The relative intensity impact of misalignment transverse vertical to the beam. [Middle] The relative intensity impact of misalignment of the guide channel pitch. [Bottom] The relative intensity impact of angular misalignment around the shutter rotation axis.
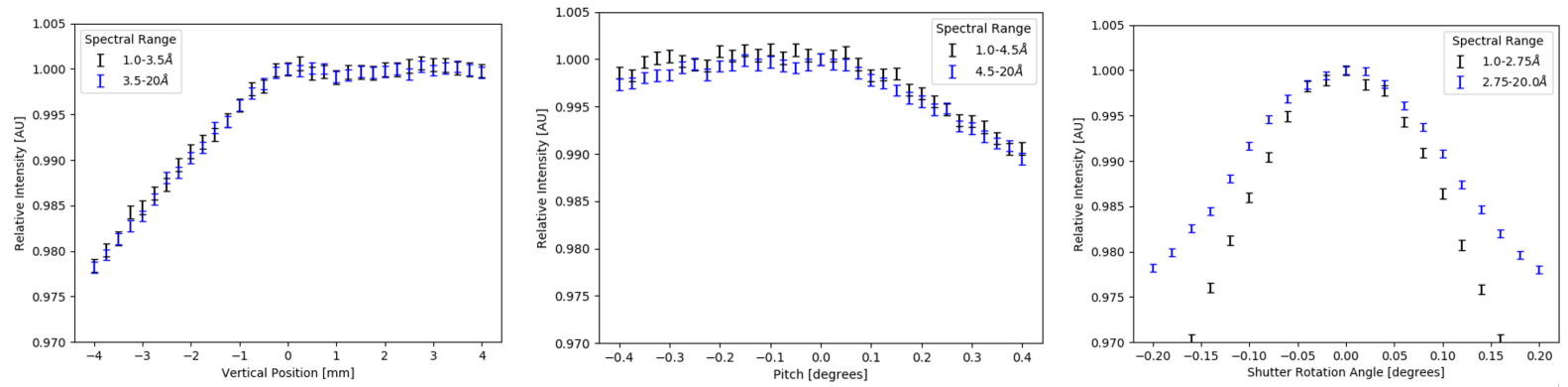

Figure 16. Plots describing misalignment effects for the NB2 Beam Guide. [Top] The relative intensity impact of misalignment transverse vertical to the beam. [Middle] The relative intensity impact of misalignment of the guide channel pitch. [Bottom] The relative intensity impact of angular misalignment around the shutter rotation axis. 

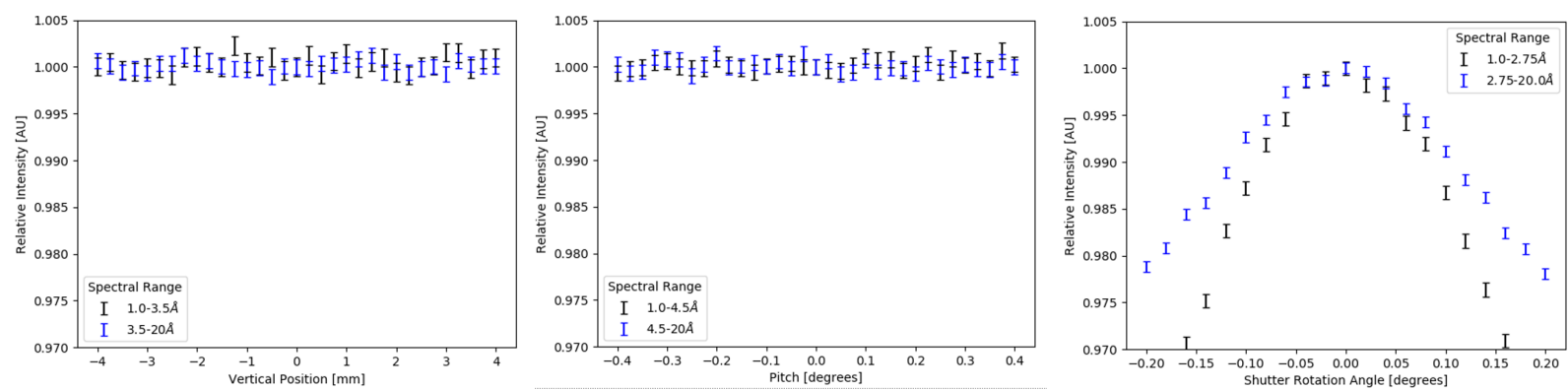

Figure 17. Plots describing misalignment effects for the NB3 Beam Guide. [Top] The relative intensity impact of misalignment transverse vertical to the beam. [Middle] The relative intensity impact of misalignment of the guide channel pitch. [Bottom] The relative intensity impact of angular misalignment around the shutter rotation axis.
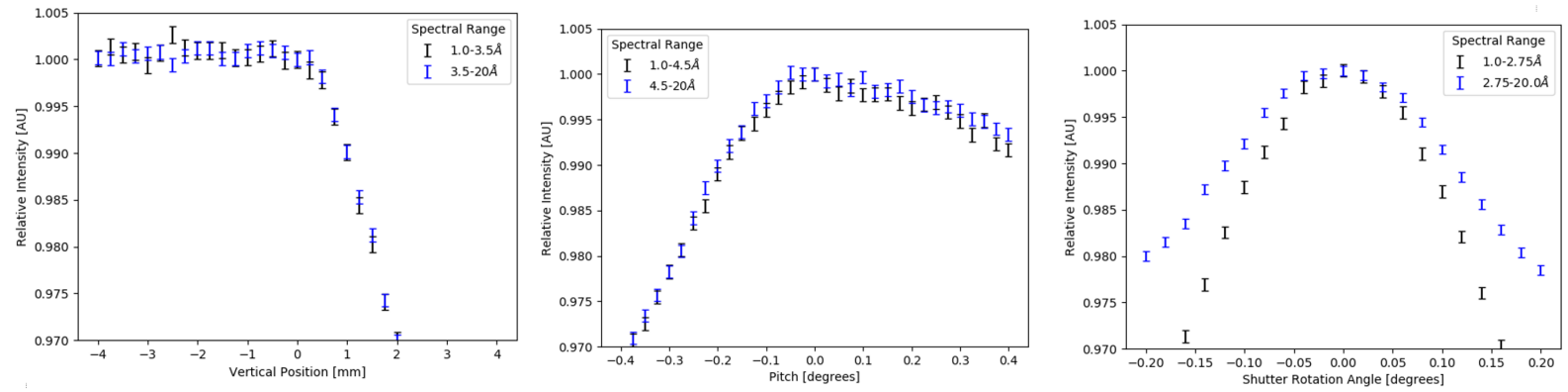

Figure 18. Plots describing misalignment effects for the NB4 Beam Guide. [Top] The relative intensity impact of misalignment transverse vertical to the beam. [Middle] The relative intensity impact of misalignment of the guide channel pitch. [Bottom] The relative intensity impact of angular misalignment around the shutter rotation axis.
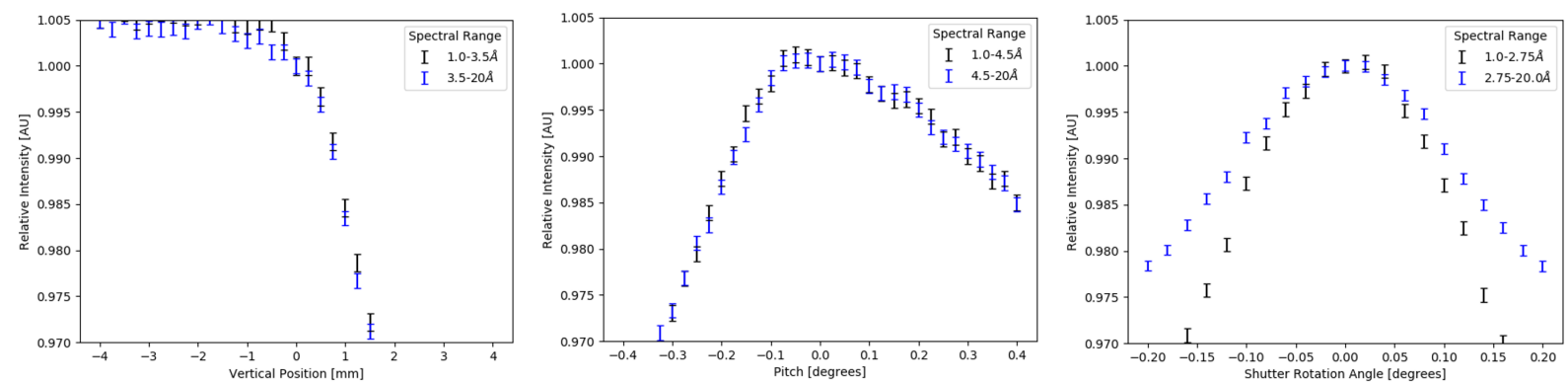

Figure 19. Plots describing misalignment effects for the NB5 Beam Guide. [Top] The relative intensity impact of misalignment transverse vertical to the beam. [Middle] The relative intensity impact of misalignment of the guide channel pitch. [Bottom] The relative intensity impact of angular misalignment around the shutter rotation axis. 

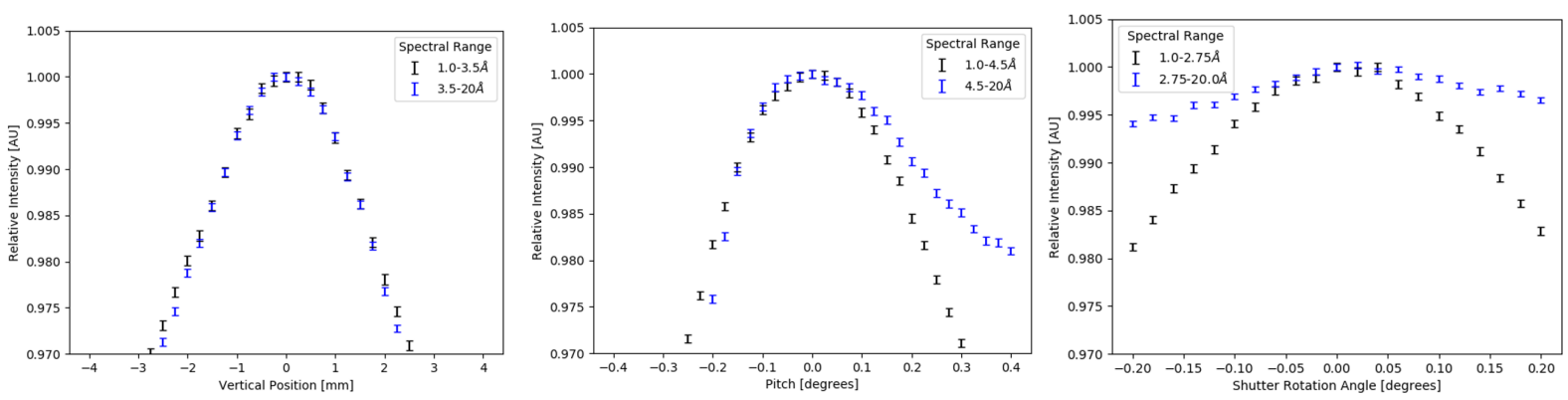

Figure 20. Plots describing misalignment effects for the NB6 Beam Guide. [Top] The relative intensity impact of misalignment transverse vertical to the beam. [Middle] The relative intensity impact of misalignment of the guide channel pitch. [Bottom] The relative intensity impact of angular misalignment around the shutter rotation axis. 


\section{SUMMARY}

A neutron optic concept of the guide channel insert proposed for the HB4 Main Shutter System was presented and guidelines for its design and alignment requirements were conveyed. Simulations were performed to provide understanding with regards to optimal geometry and coating selection for each surface as well as the impact of misalignment those surfaces can have on the final instrument performance. In general, it seems that alignment of these surfaces to within $+/-0.25$ millimeters and $+/-0.05^{\circ}$ across all dimensions will ensure losses are no worse than $1 \%$ for any of the guide starts. A more specific quantification showing the impacts on each instrument in terms of relative loss per unit can be seen in Table 4. The inverse of the these values define the alignment range required to maintain losses below $1 \%$.

Table 4. A table of values interpreting the simulated losses and plots for each beam guide. Each beam guide simulation provided a plot of intensity losses across each dimension range. Taking the average loss over that scanned range provides a percent-loss-per-unit, where the unit is either millimeters or degrees. This value is then inverted to provide upper and lower misalignment limits for each dimension, assuming a loss of $1 \%$ can be tolerated.

\begin{tabular}{|c|c|c|c|c|c|c|c|c|c|c|c|c|}
\hline \multirow{2}{*}{ Beam $\backslash$ Dimension } & \multicolumn{2}{|c|}{$\mathbf{x}[\% / \mathbf{m m}]$} & \multicolumn{2}{|c|}{$\mathrm{y}[\% / \mathbf{m m}]$} & \multicolumn{2}{|c|}{$\mathrm{z}[\% / \mathbf{m m}]$} & \multicolumn{2}{|c|}{$\alpha[\% / \mathrm{deg}]$} & \multicolumn{2}{|c|}{$\beta[\% / \mathrm{deg}]$} & \multicolumn{2}{|c|}{$\gamma[\% /$ deg $]$} \\
\hline & lower & upper & lower & upper & lower & upper & ower & upper & lower & upper & lower & upper \\
\hline NB1 & 2.25 & 2.25 & 1.06 & 0.00 & 0.01 & 0.30 & 4.75 & 5.25 & 15.00 & 15.00 & 2.50 & 3.13 \\
\hline NB2 & 2.25 & 2.25 & 0.53 & 0.00 & 0.01 & 0.30 & 0.00 & 0.25 & 18.75 & 18.75 & 2.50 & 3.13 \\
\hline NB3 & 2.25 & 2.25 & 0.01 & 0.01 & 0.01 & 0.30 & 0.01 & 0.01 & 18.75 & 18.75 & 2.50 & 3.13 \\
\hline NB4 & 2.25 & 2.25 & 0.01 & 1.50 & 0.01 & 0.30 & 7.90 & 1.83 & 18.75 & 18.75 & 2.50 & 3.13 \\
\hline NB5 & 2.25 & 2.25 & 1.50 & 0.01 & 0 & 0.30 & 9.37 & 3.75 & 18.75 & 18.75 & 2.50 & 3.13 \\
\hline NB6 & 2.25 & 2.25 & 1.00 & 1.00 & 0.01 & 0.30 & 11.00 & 11.00 & 10.00 & 10.00 & 2.50 & 3.13 \\
\hline MAXI & 2.25 & 2.25 & 1.50 & 1.50 & 0.01 & 0.30 & 11.00 & 11.00 & 18.75 & 18.75 & 2.50 & 3.13 \\
\hline Tolerance & \multicolumn{2}{|c|}{$\mathbf{x}[\mathbf{m m} / \%]$} & \multicolumn{2}{|c|}{$\mathrm{y}[\mathrm{mm} / \%]$} & \multicolumn{2}{|c|}{$\mathrm{z}[\mathrm{mm} / \%]$} & \multicolumn{2}{|c|}{$\alpha[\operatorname{deg} / \%]$} & \multicolumn{2}{|c|}{$\beta[\operatorname{deg} / \%]$} & \multicolumn{2}{|c|}{$\gamma[\operatorname{deg} / \%]$} \\
\hline $\begin{array}{c}\text { (mm or deg) for } \\
1 \% \text { loss }\end{array}$ & $\mathbf{0}$ & .44 & 0.67 & 0.67 & 100.0 & 3.33 & 0.09 & 0.09 & 0.05 & 0.05 & 0.40 & 0.32 \\
\hline
\end{tabular}




\section{REFERENCES}

\section{References}

[1] Georg Ehlers, Matthew J. Frost, Garrett E. Granroth, Thomas Huegle, Richard M. Ibberson, and J. Lee Robertson. A Replacement Cold Neutron Guide System for HFIR (Conceptual Design Report). Technical Report ORNL/TM-2020/1568, Oak Ridge National Lab. (ORNL), Oak Ridge, TN (United States), July 2020.

[2] Peter Kjaer Willendrup and Kim Lefmann. McStas (ii): An overview of components, their use, and advice for user contributions. Journal of Neutron Research, 23(1):7-27, April 2021.

[3] Franz X. Gallmeier and Igor Remec. HFIR Cold Source Upgrade Options. Technical Report ORNL/TM-2018/820, Oak Ridge National Lab. (ORNL), Oak Ridge, TN (United States), September 2018.

[4] J. L. Robertson and E. B. Iverson. Measurement of the neutron spectrum of the hb-4 cold source at the high flux isotope reactor at oak ridge national laboratory. In Reactor Dosimetry State of the Art 2008, pages 85-93. WORLD SCIENTIFIC, August 2009.

[5] J. A. Bucholz. Physics Analyses in the Design of the HFIR Cold Neutron Source. Technical Report ORNL/CP-104770, Oak Ridge National Lab. (ORNL), Oak Ridge, TN (United States), September 1999. 

APPENDIX 


\section{A CORRECTION TO 2007 HB4 COLD SOURCE BRIGHTNESS MEASUREMENT}

The first brightness measurement of the refurbished cold source in 2007 utilized a TOF instrument setup that allowed for precise determination of the neutron brightness spectrum from $0.7 \AA$ to $10.0 \AA$ [4]. The apparatus used a disk chopper, a neutron detector and a data acquisition system that recorded detector pulses relative to the disk chopper opening trigger time. The result was a well resolved TOF spectrum expected of a $22.5 \mathrm{~K}$ cold source. In order to accurately quantify the brightness of the source, determination of the chopper duty cycle, detector efficiency and aperture geometry is required. The aperture geometry (and thus the acceptance correction to determine the true brightness) was "difficult to calculate analytically because the collimation system includes both rectangular and circular apertures." An estimate of the acceptance was attempted by approximating the round apertures with square apertures of equivalent side-length. Using this estimate the overall acceptance in the detector was determined to be $6.8 \times 10^{-6}$ $\mathrm{cm}^{2} \cdot$ sr. Based on this acceptance, a triple Maxwell-Boltzmann distribution can be used to replicate the cold source spectrum brightness for instrument simulation purposes. The neutron wavelength spectral shape is

$$
B(\lambda)=\sum_{i=1}^{3} 2 I_{i} \frac{a_{i}^{2}}{\lambda^{5}} e^{-a_{i} / \lambda^{2}} \quad a_{i}=\frac{949.0}{T_{i}}
$$

The parameters that best fit this data are seen in Figure 21. Using a McStas simulation replicating the
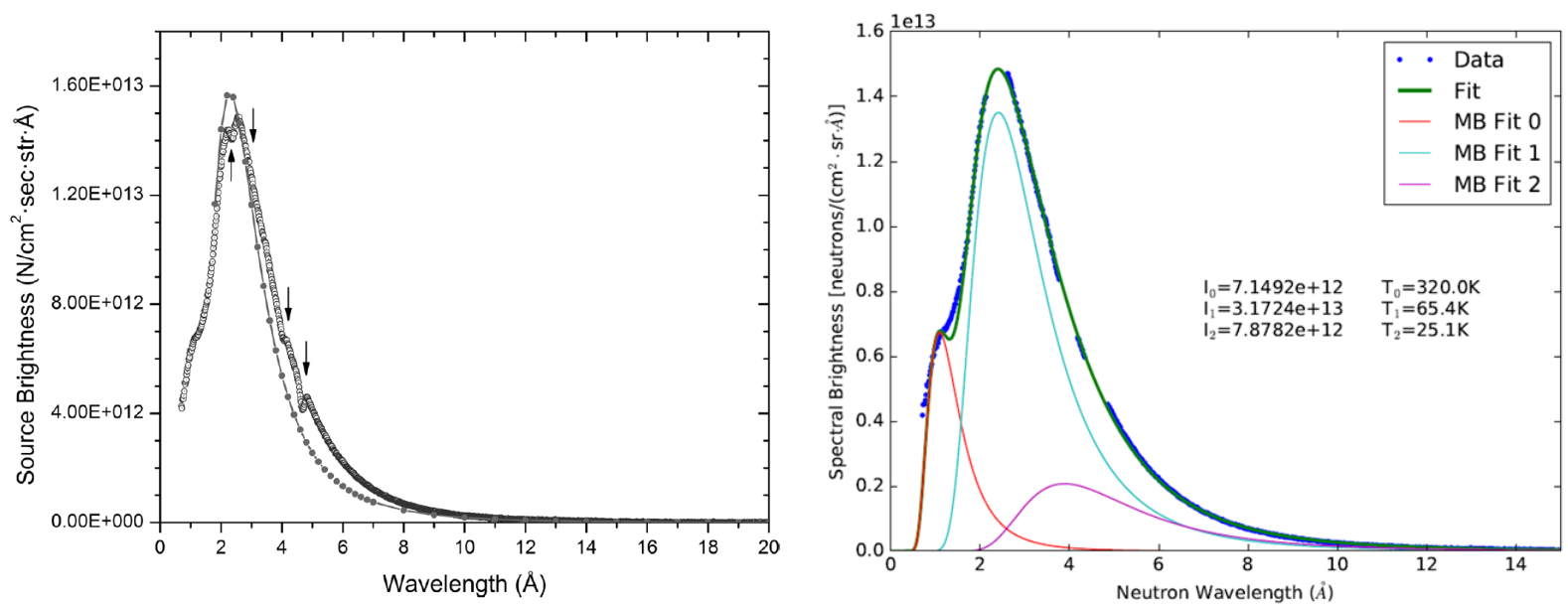

Figure 21. The simulated, measured and fitted brightness from the HFIR cold source in 2007. [Left]

The spectrum as measured and as simulated using MCNP computer code. [Right] a triple Maxwell-Boltzmann fit to the corrected data taken during the testing.

described 2007 instrument configuration, one should be able to confirm the brightness values provide the expected corrected brightness at the detector based on what is known about the geometry of the apertures in series (acceptance). However, an attempted replication of this result is seen in Figure 22, and the simulated result appears to be about $15 \%$ higher than expected when using the parameters fitted to the data provided from 2007.

Maintaining confidence in the standard McStas component Source_gen, one assumes that a correction is needed in the acceptance factor of the data normalization, rather than there being an issue with the source component itself. In order to determine the actual acceptance of the instrument used to do the measurement 

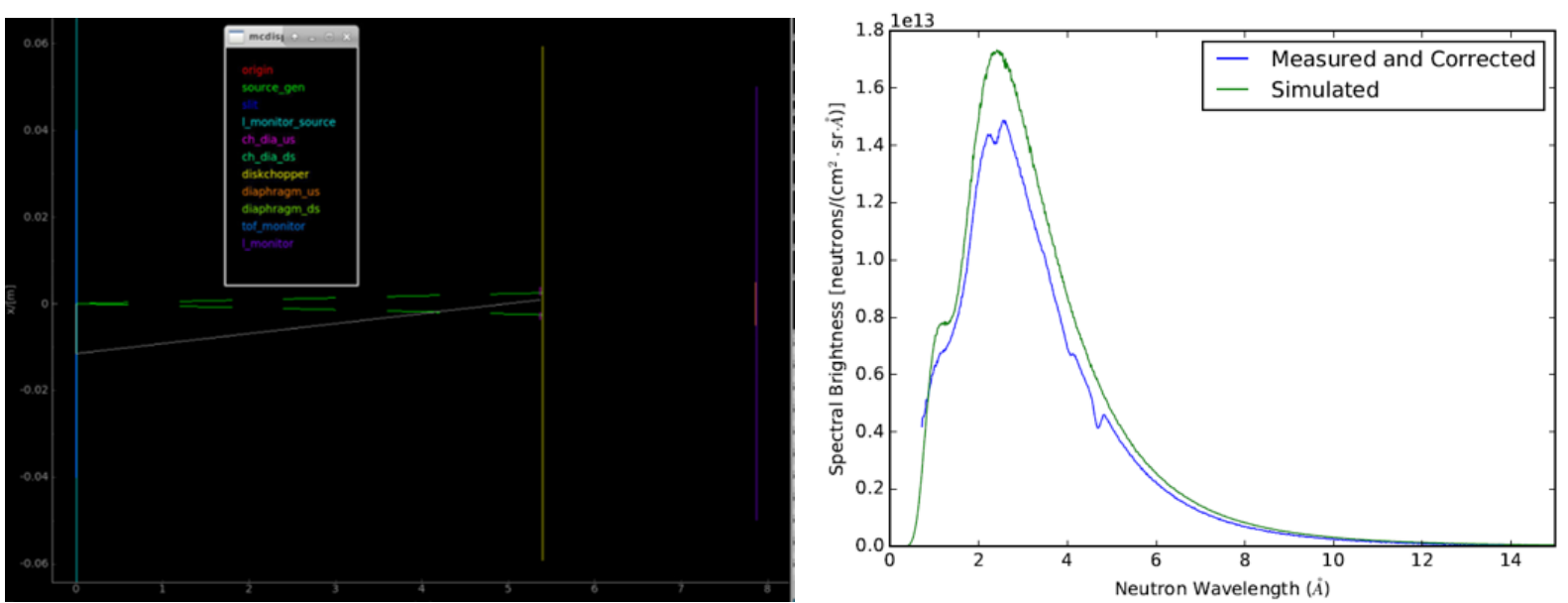

Figure 22. A schematic describing the layout of the brightness measurement and associated spectrum. [Left] The simulated TOF instrument to observe the spectrum of the cold source. [Right] The resulting brightness spectrum from the measurement and the simulated one spectrum using the parameters from Figure 21.

a Monte Carlo method much like that could be used to determine the value of $\pi$ is utilized. In this case, one samples the full range of phase space at the source that will fully illuminate the aperture used to define the view of the source at the detector. As seen in figure 23, the whole range of angles $\vec{\theta}$ is sampled across the whole range of positions $\vec{x}$. Only a subset of those positions and trajectories will be transmitted through the aperture system and onto the detector. Thus, if one knows the full phase space emmittance range from the source $E_{S}$, the acceptance of the system $A$ is the ratio of detected events $N^{\prime}$ to sampled events $N$ times that source emmitance.

$$
A=\frac{N^{\prime}}{N} E_{S}
$$

Using the McStas instrument definition found at https://code.ornl.gov/sns-neutronics/mcstas-wg /hb4-cold-source/-/blob/master/mcstas/chopped_brightness_measurement_2007.instr, one is able to quantify to a reasonable precision $(\sim 2 \%)$ the acceptance of the measurement setup to be $7.95 \times 10^{-6}$ $\mathrm{cm}^{2} \cdot \mathrm{sr}$. This is a $17 \%$ increase in the acceptance used in the original 2007 report, thus revising the expected brightness values downwards by the same amount.

These new values should be used for any instrument simulation work involving the current HB4 Cold Source design at $85 \mathrm{MW}$, and can be seen in Table 1. 

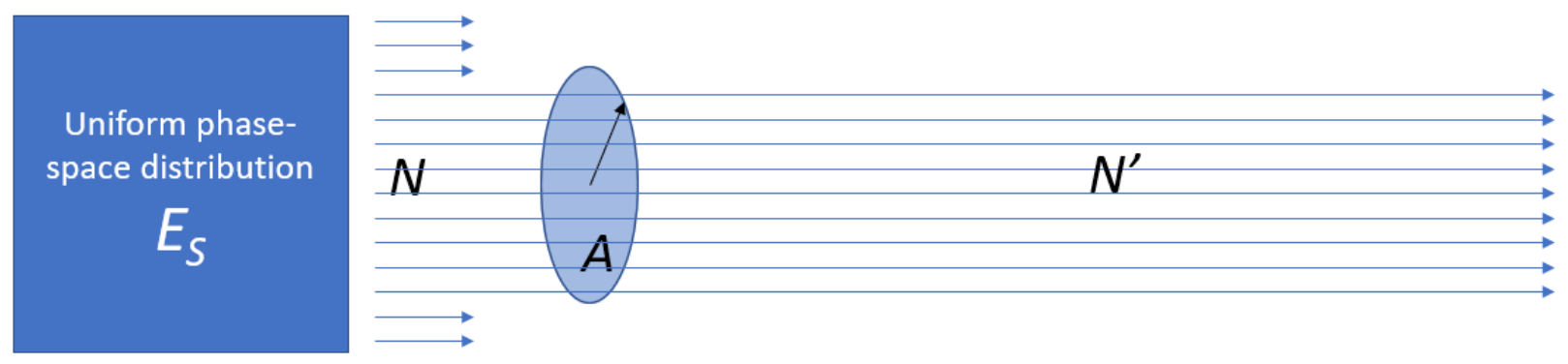

Figure 23. A schematic describing acceptance determination via Monte Carlo simulation. The acceptance is the ratio of successful particle trajectories to the number created at the source and times the known emmitance range of the source. 

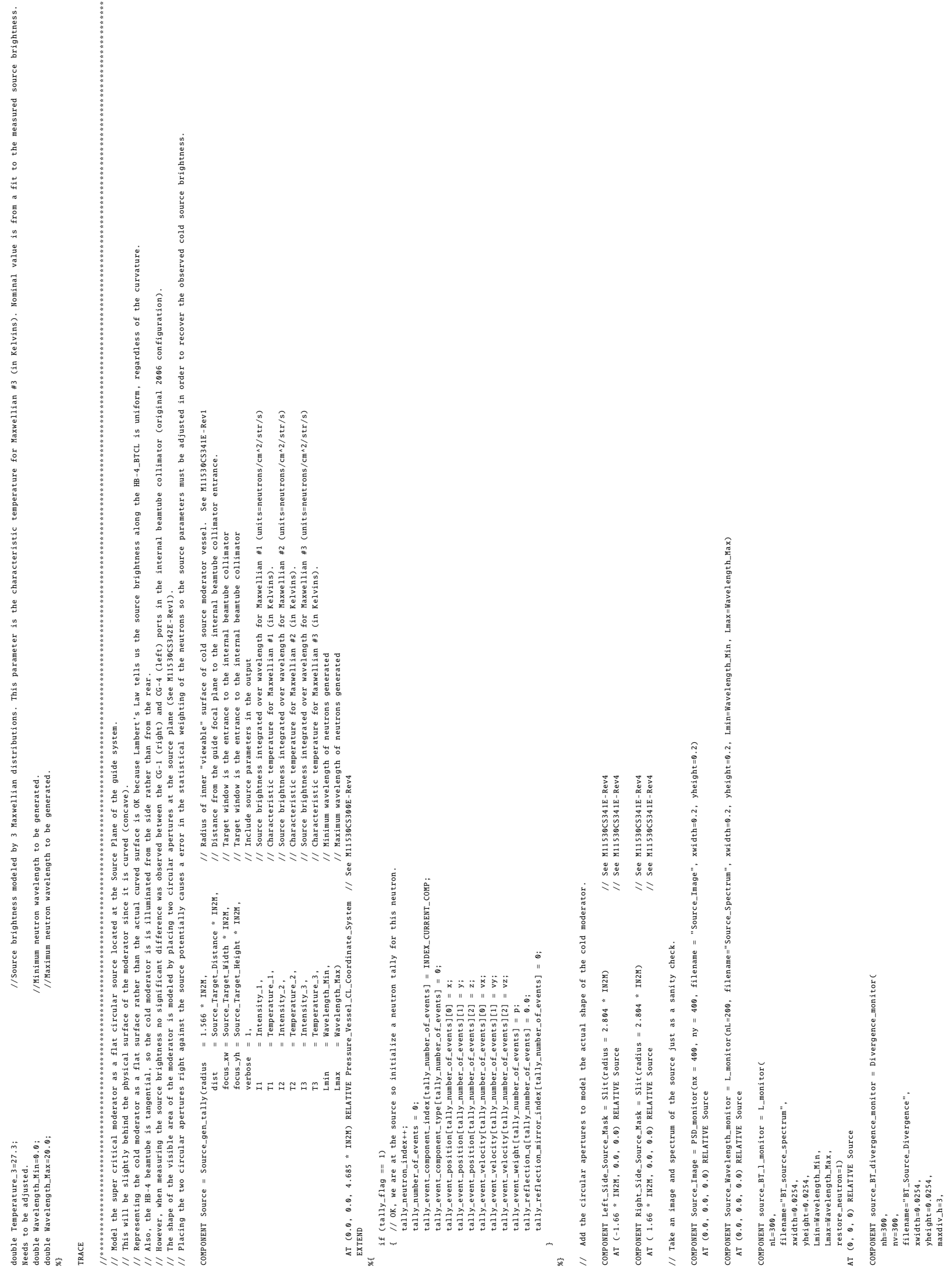


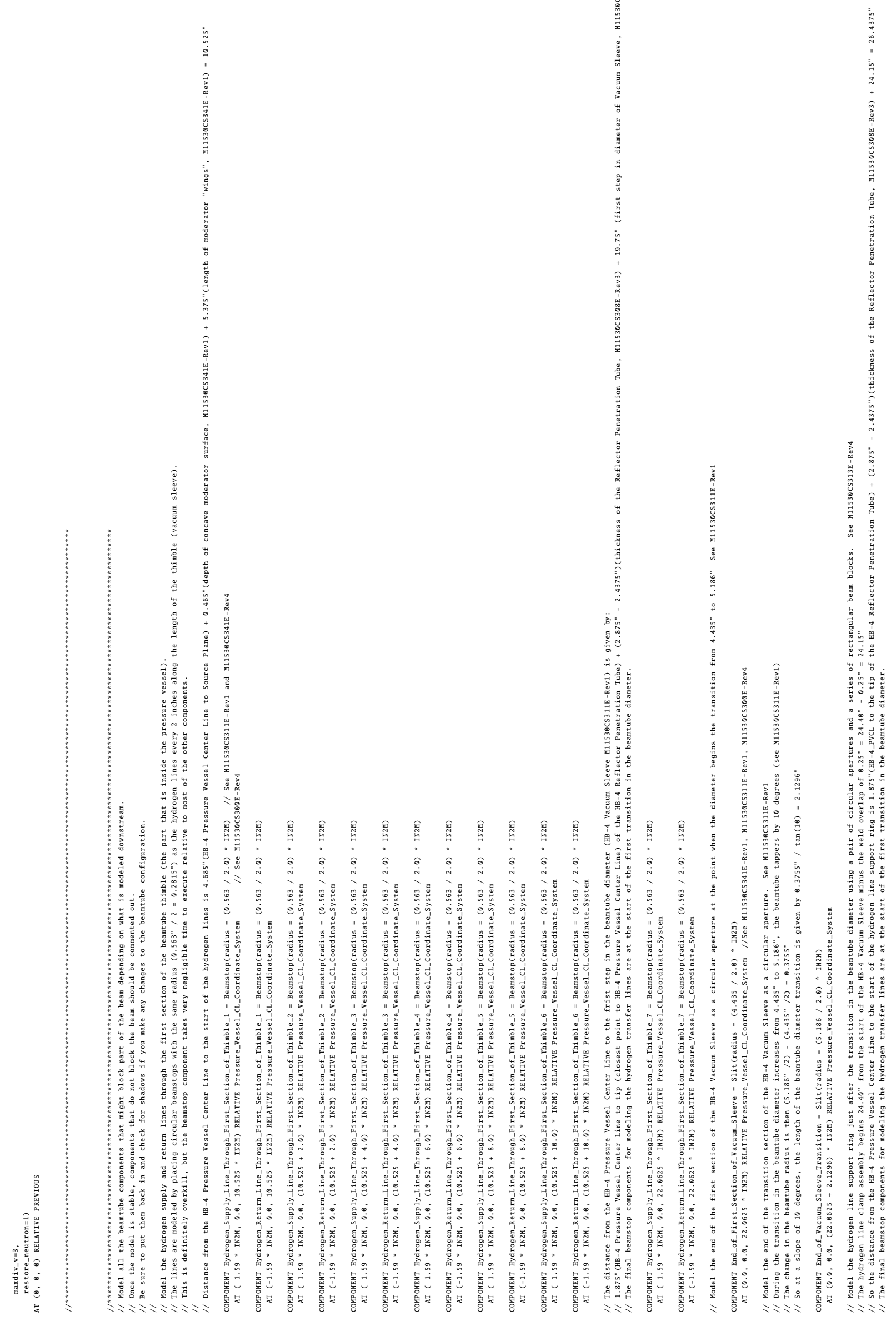




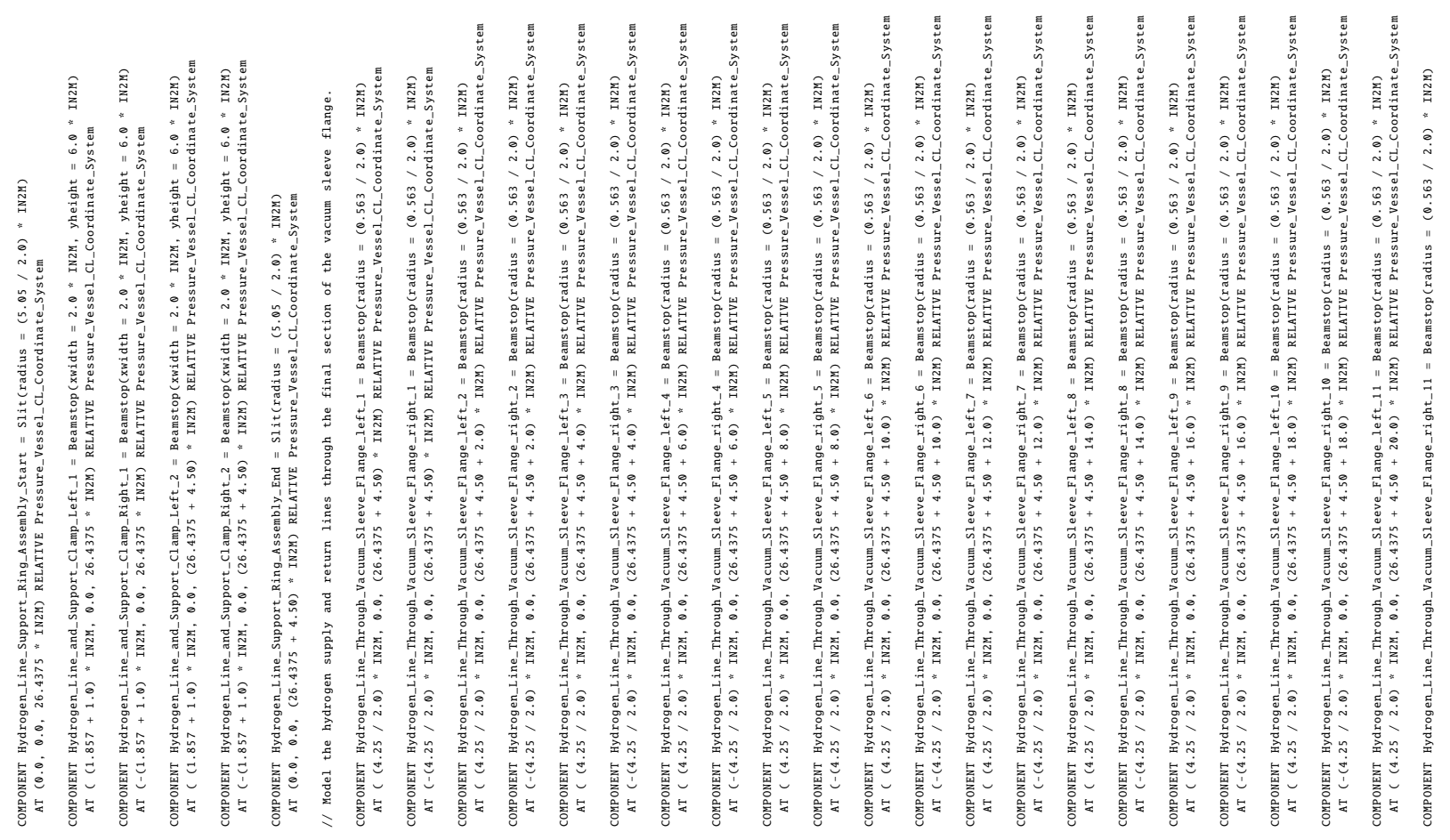




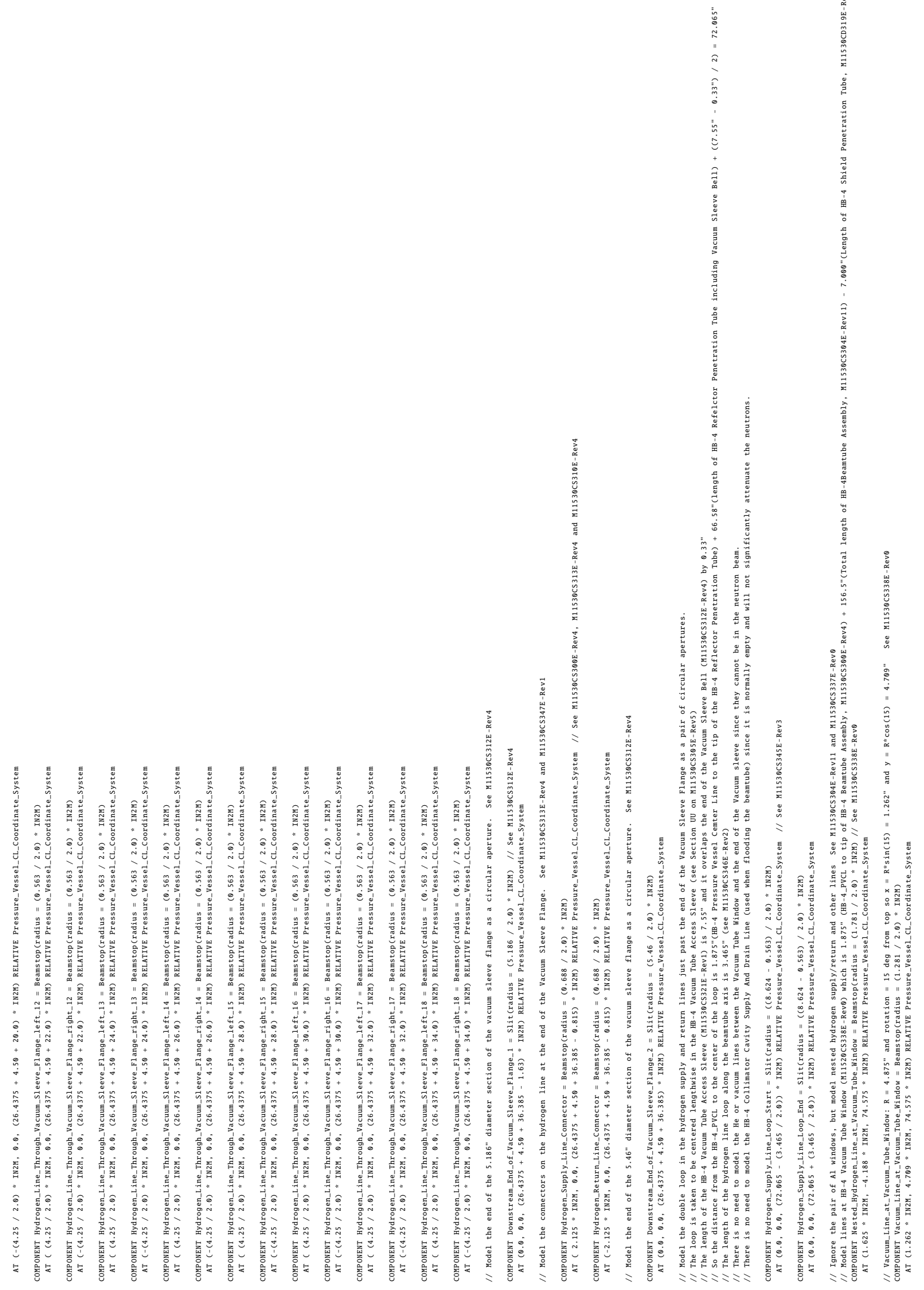




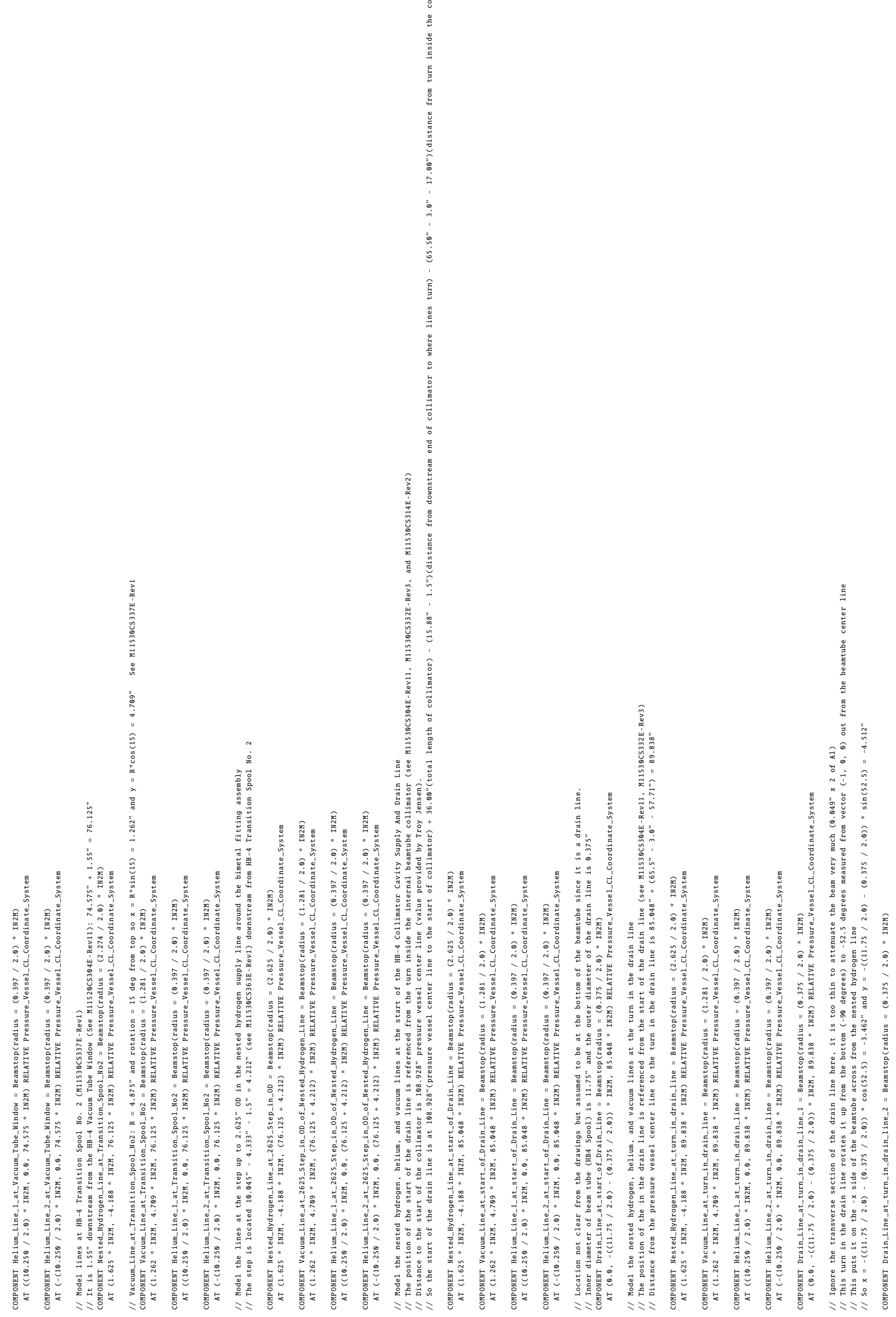




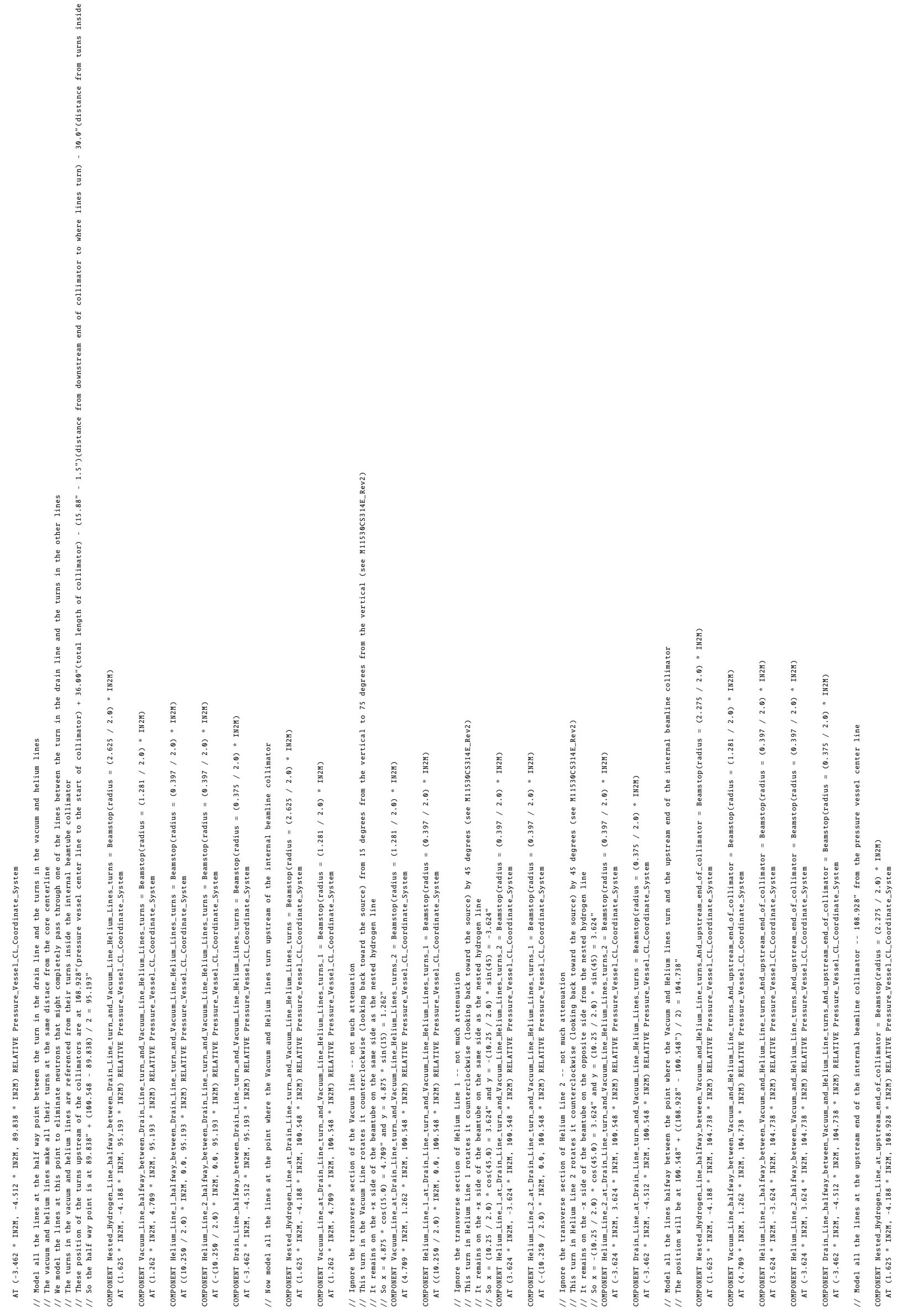




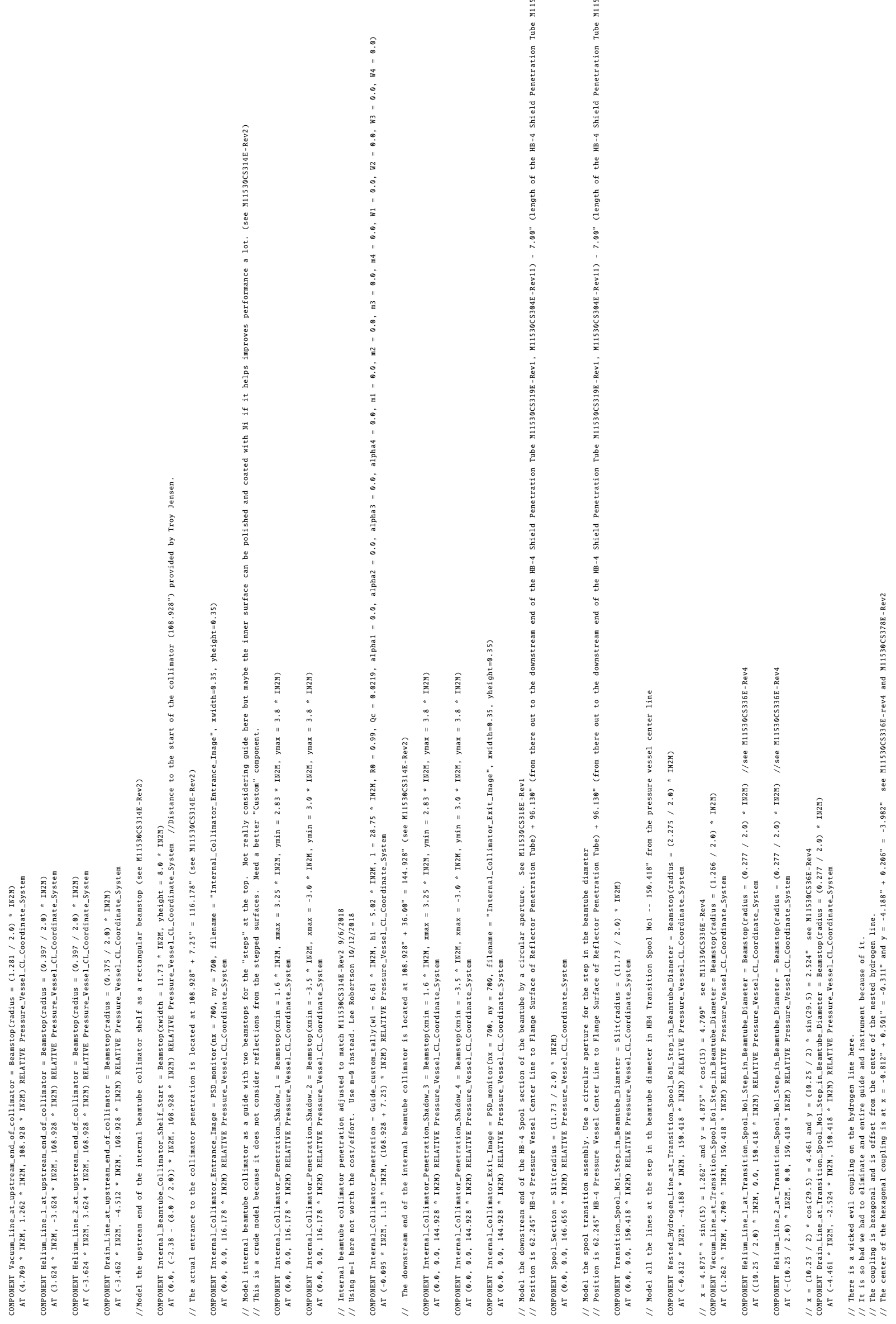




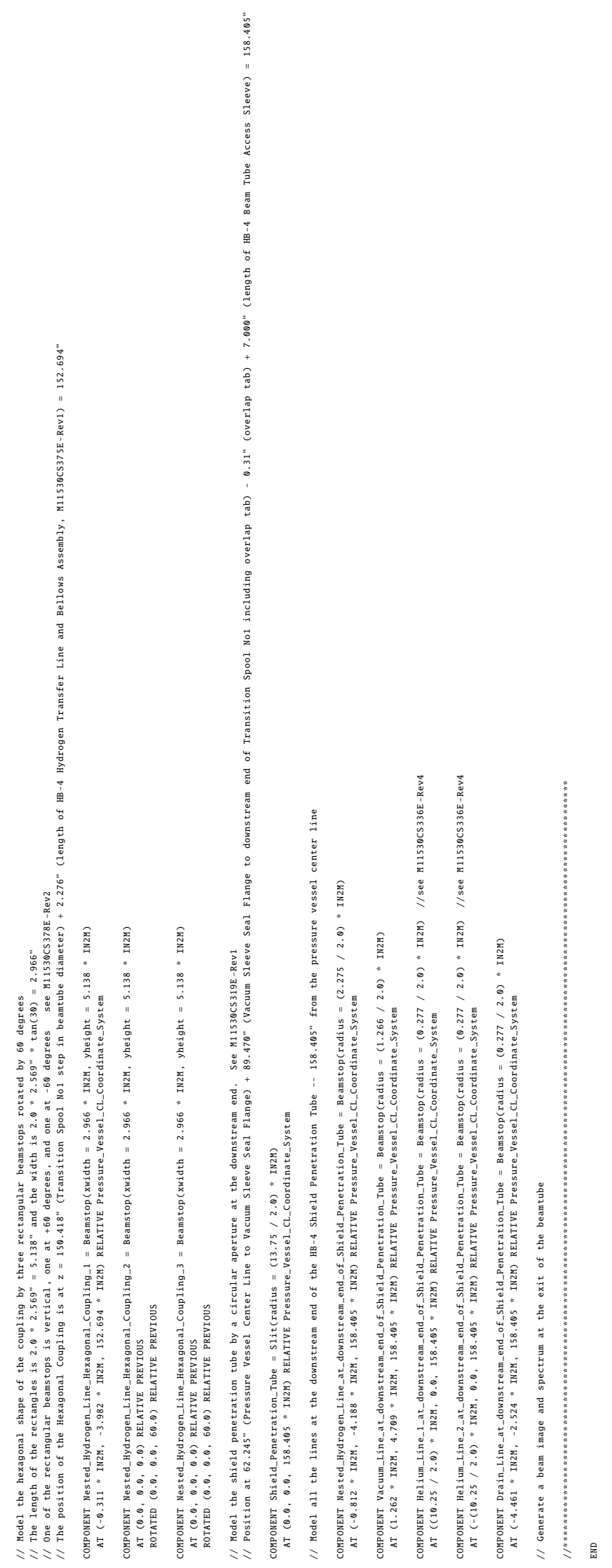

A-13 


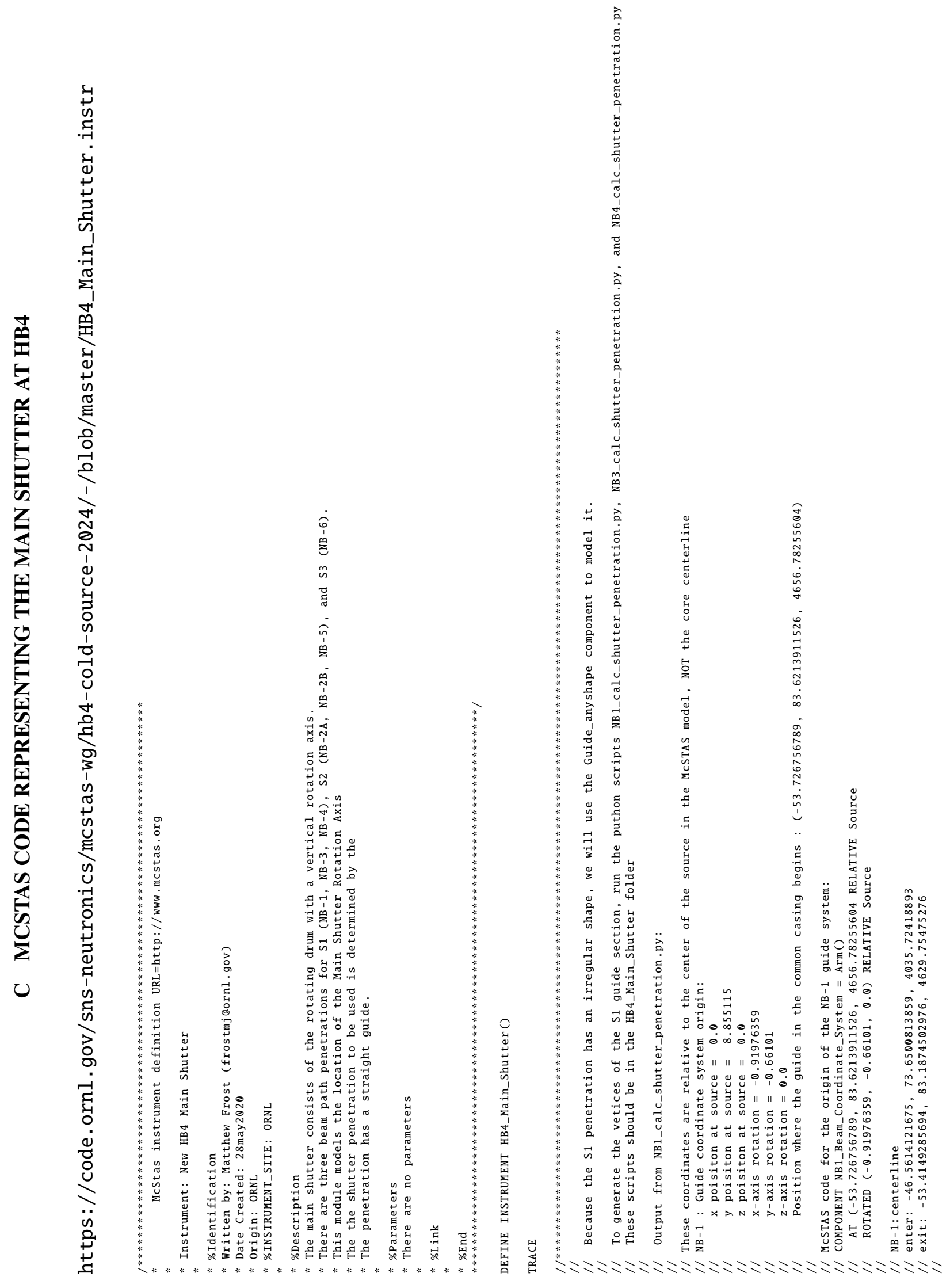




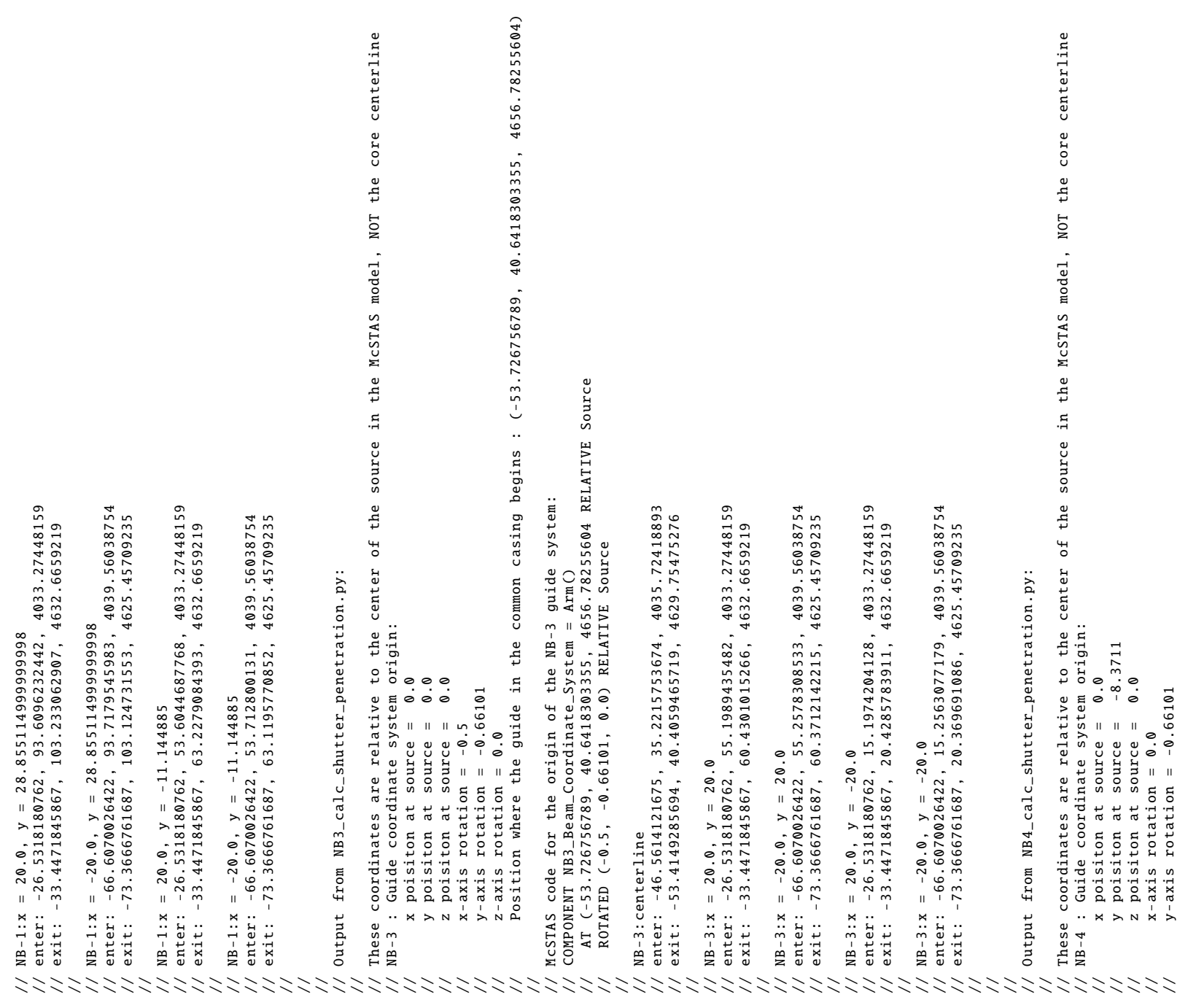




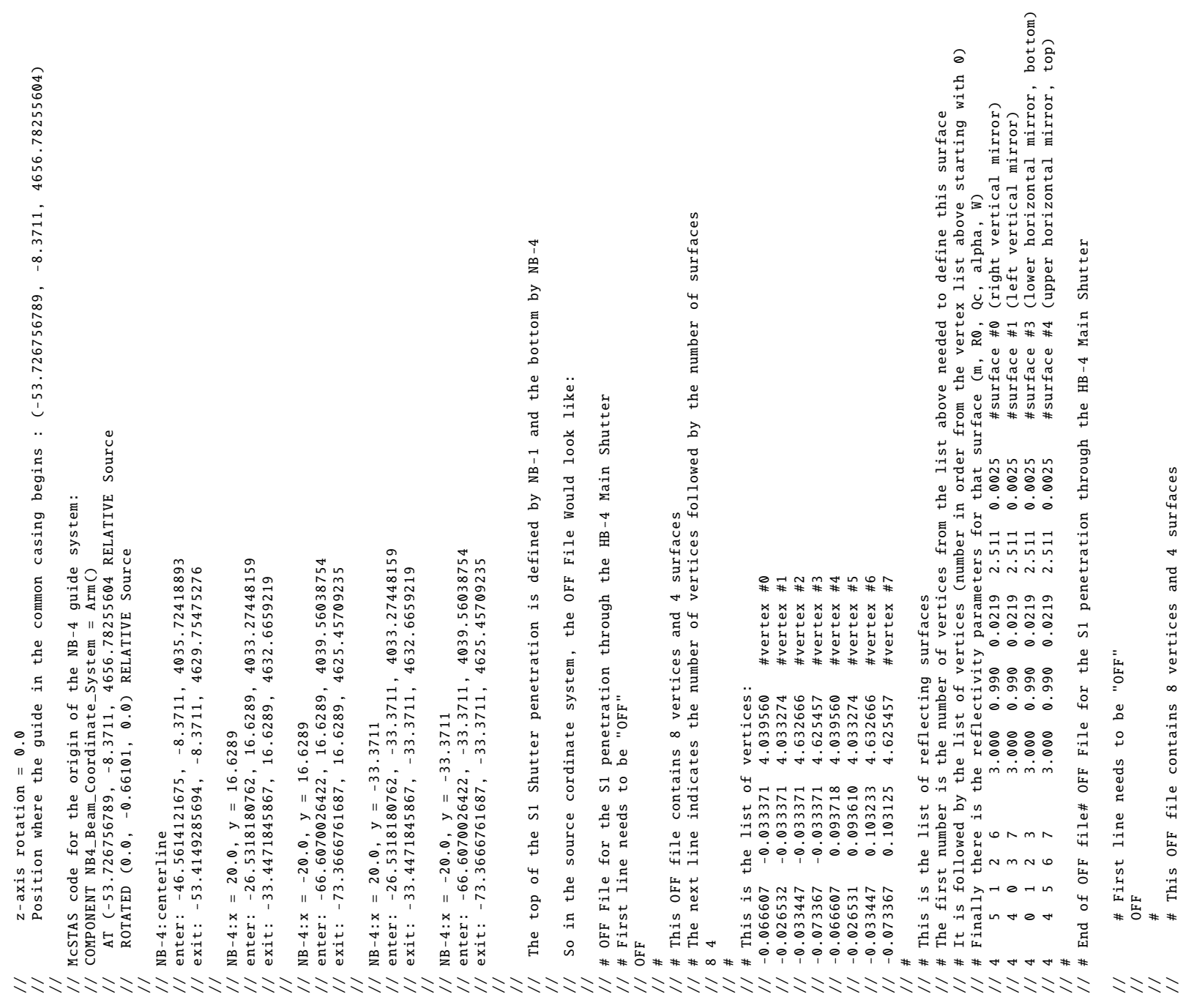




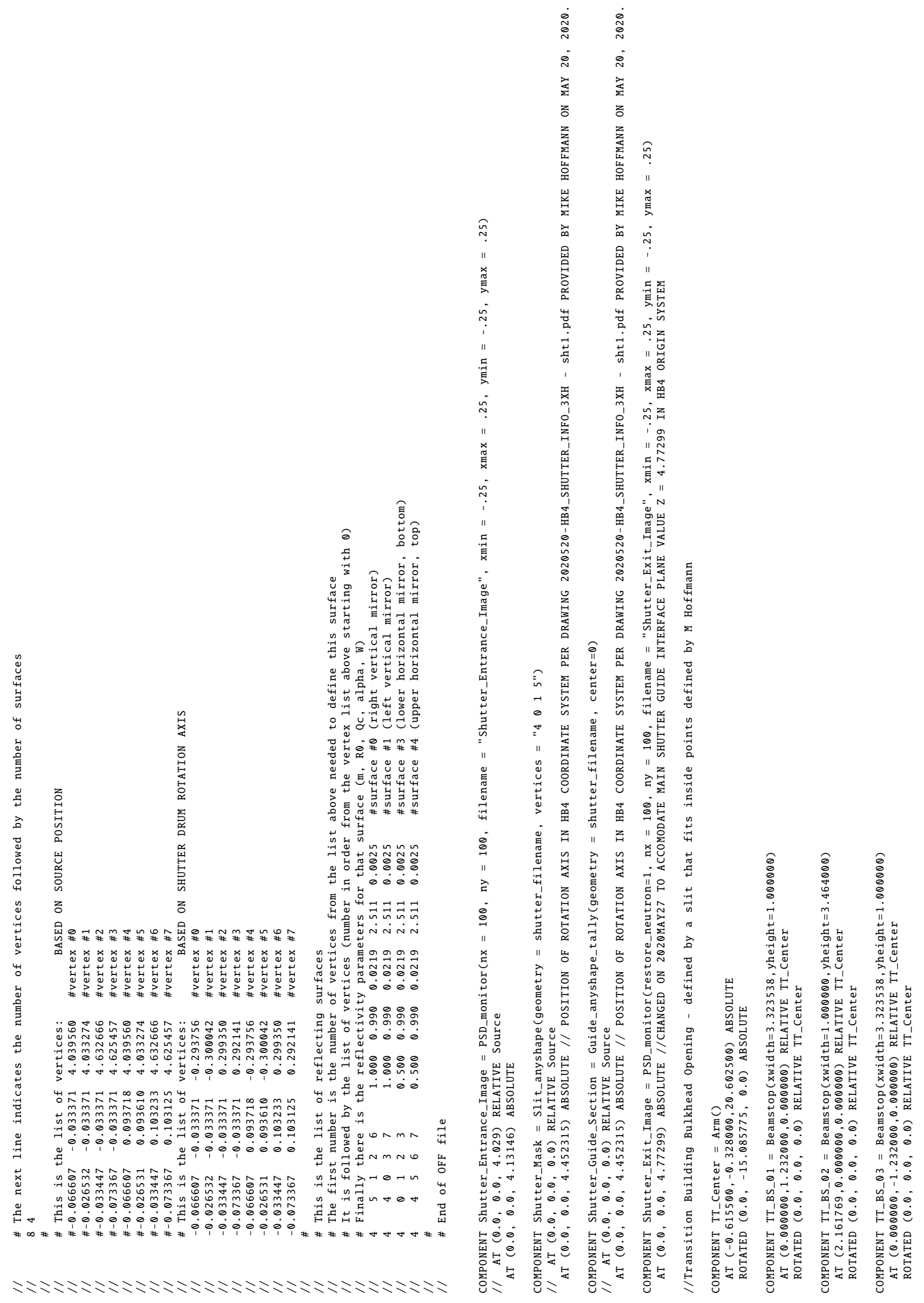




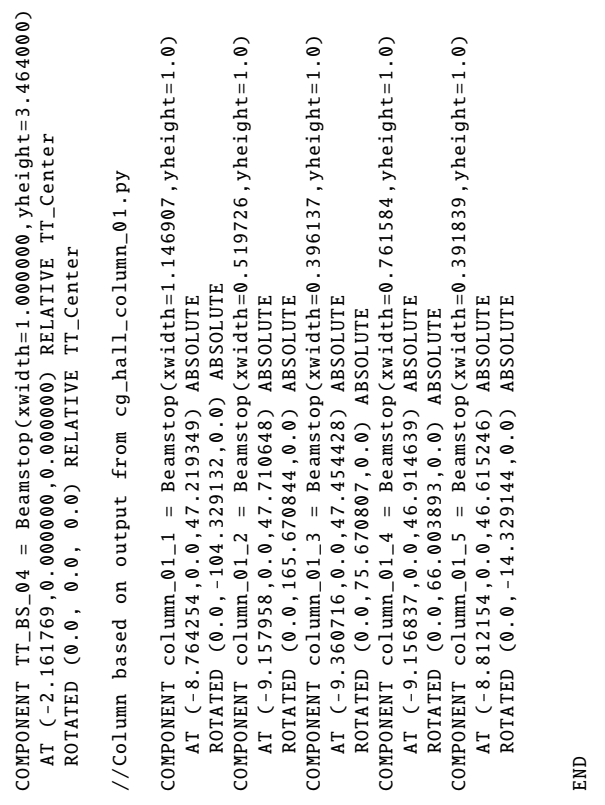

A-18 


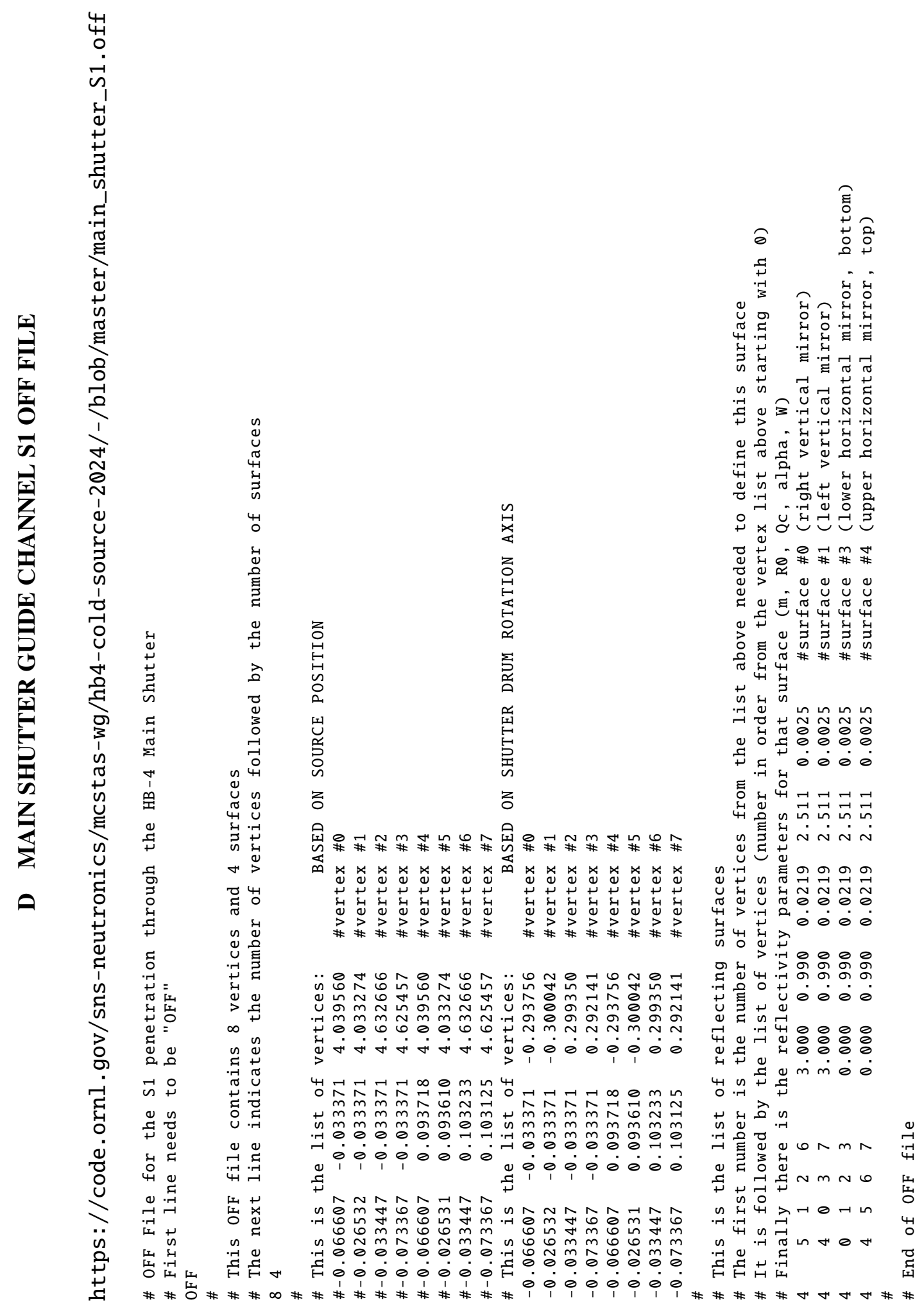




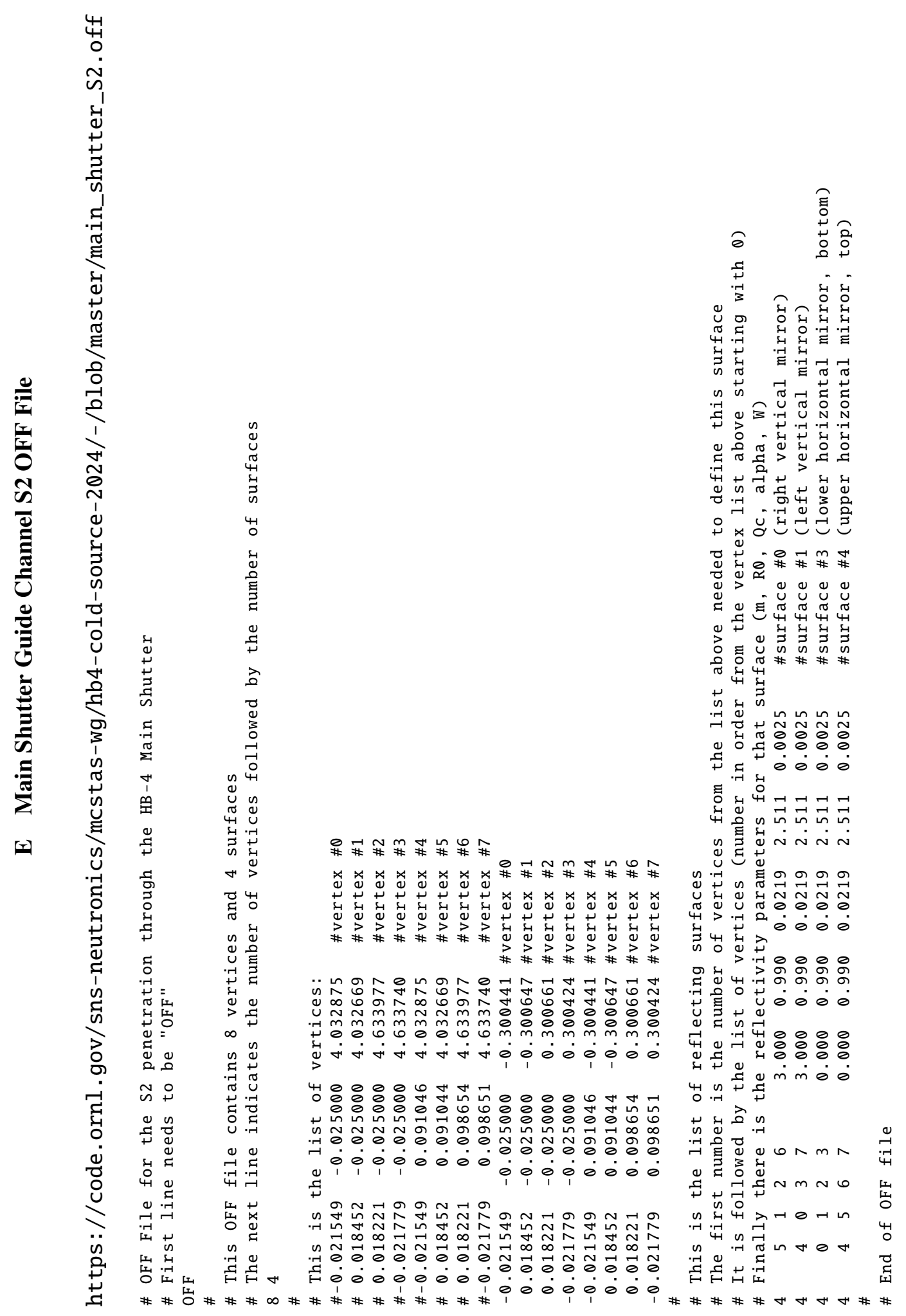




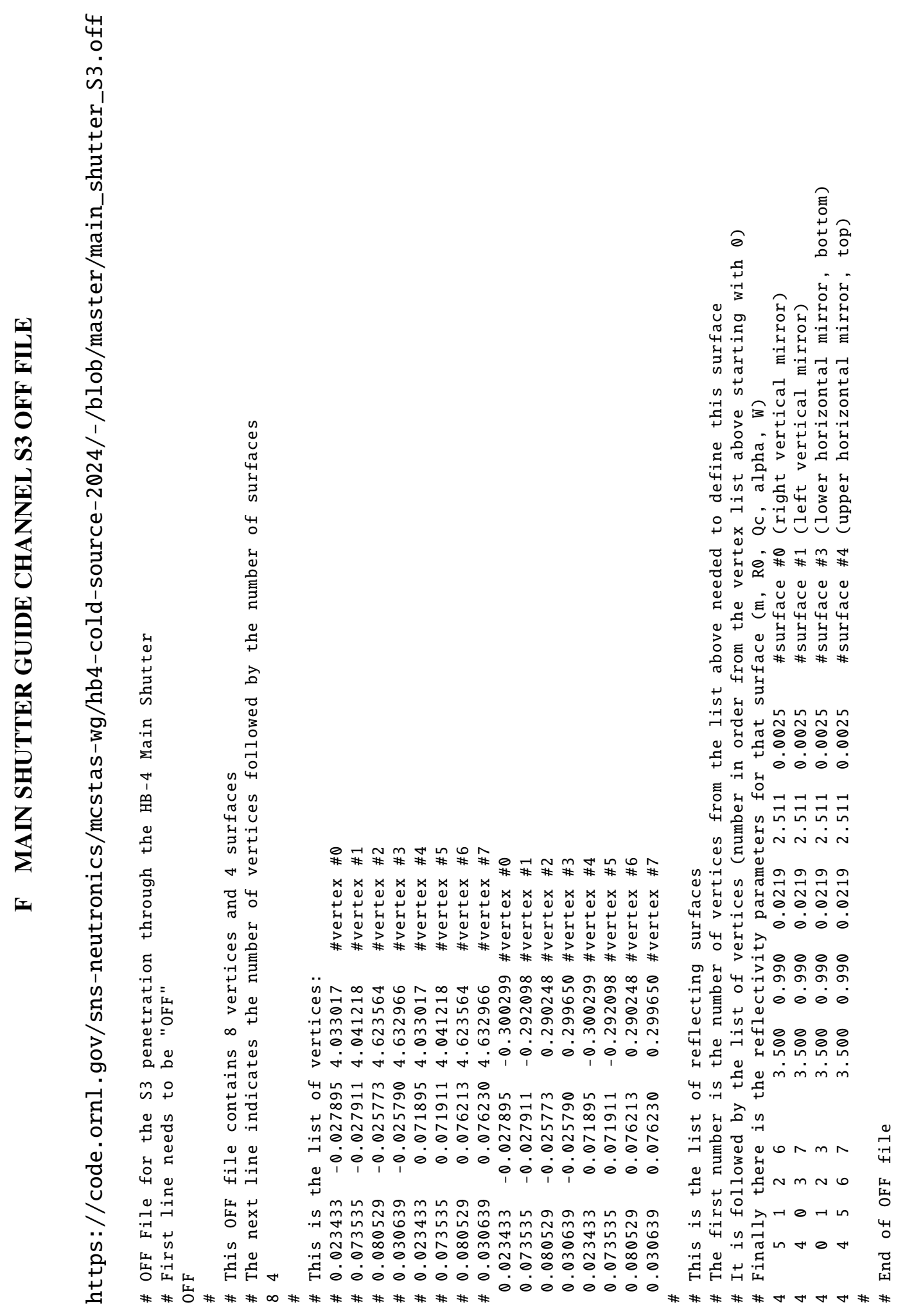





(n)

